\title{
Efficient Fast Stereo Acoustic Echo Cancellation Based on Pairwise Optimal Weight Realization Technique
}

\author{
Masahiro Yukawa, Noriaki Murakoshi, and Isao Yamada \\ Department of Communications and Integrated Systems, Graduate School of Science and Engineering, Tokyo Institute of Technology, \\ 2-12-1 Ookayama, Meguro-Ku, Tokyo 152-8550, Japan
}

Received 1 February 2005; Revised 1 October 2005; Accepted 4 October 2005

\begin{abstract}
In stereophonic acoustic echo cancellation (SAEC) problem, fast and accurate tracking of echo path is strongly required for stable echo cancellation. In this paper, we propose a class of efficient fast SAEC schemes with linear computational complexity (with respect to filter length). The proposed schemes are based on pairwise optimal weight realization (POWER) technique, thus realizing a "best" strategy (in the sense of pairwise and worst-case optimization) to use multiple-state information obtained by preprocessing. Numerical examples demonstrate that the proposed schemes significantly improve the convergence behavior compared with conventional methods in terms of system mismatch as well as echo return loss enhancement (ERLE).
\end{abstract}

Copyright (c) 2006 Masahiro Yukawa et al. This is an open access article distributed under the Creative Commons Attribution License, which permits unrestricted use, distribution, and reproduction in any medium, provided the original work is properly cited.

\section{INTRODUCTION}

The ultimate goal of this paper is to develop an efficient adaptive filtering scheme, with linear computational complexity, to stably cancel acoustic coupling, from loudspeakers to microphones, occurring in telecommunications with stereophonic audio systems. This acoustic coupling is commonly called acoustic echo (we just call it echo in the following). The stereophonic acoustic echo cancellation (SAEC) problem has become a central issue when we design high-quality, handsfree, and full-duplex systems (e.g., advanced teleconferencing, etc.) [1-13]. A direct application of a monaural echo cancelling algorithm to SAEC usually results in unacceptably slow convergence [1-3], and this phenomenon is mathematically clarified in [5], showing that the normal equation to be solved for minimization of residual echo is often illconditioned or has infinitely many solutions due to inherent dependency caused by highly cross-correlated stereo input signals (see Section 2.2).

Decorrelation of the inputs is a pathway to fast and accurate tracking of echo paths (impulse responses), which is necessary for stable echo cancellation $[6,8,14,15]$. A great deal of effort has been devoted to devise preprocessing of the inputs [3, 5, 14-22] (see Appendix A). In other words, these preprocessing techniques relax the ill-conditioned situation with use of additional information provided artificially by feeding less cross-correlated input signals. Based on the preprocessing [5], real-time SAEC systems have been effectively implemented, for example, in $[8,13]$. Under rapidly time-varying situations, however, further convergence acceleration is strongly required. Unfortunately, an increase of decorrelation effects by preprocessing may cause audible acoustic distortion or loss of stereo sound effects, thus the preprocessing is strictly restricted to only slight modification of the input signal. The remaining major challenges in SAEC with preprocessing are twofold: (i) fast tracking of the echo paths within the above restriction on audio effects and (ii) low computational complexity due to necessity to adapt 4 echo cancelers with a few thousand taps [7] (see Figure 1). Now, the time is ripe to move from the early stage of devising preprocessing techniques to the next stage: utilize the additional information provided by preprocessing to the fullest extent possible.

Effective utilization of the additional information is a key to achieve the goal shown in the beginning of this introduction. We formulate the SAEC problem as a time-varying settheoretic adaptive filtering, that is, approximate the estimandum $\mathbf{h}^{*}$ (system to be estimated, true echo paths) as a point in the intersection of multiple closed convex sets that are defined with observable data and contain $\mathbf{h}^{*}$ with high probability (see Section 3.1). As a preliminary step [23], we found a clue to maximally utilize the information given by the preprocessing $[14,15]$. The preprocessing in $[14,15]$ alternately generates certain two states of inputs (see Appendix A) and it 


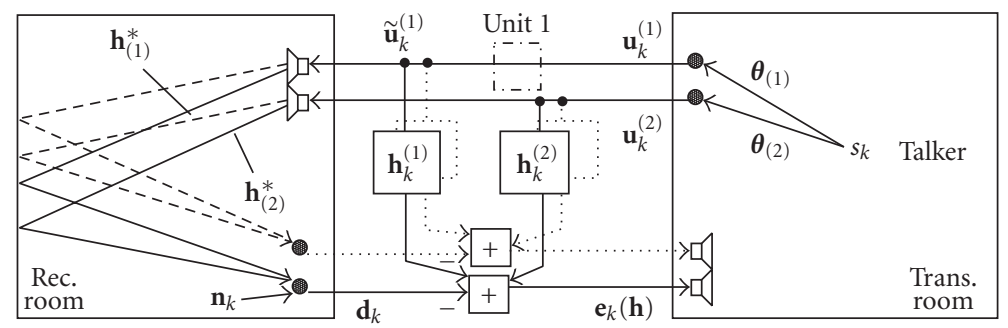

FIGURE 1: Stereophonic acoustic echo cancelling scheme; unit 1 is a preprocessing unit (see Appendix A). Note that the system is not limited to this special structure but can be any appropriate structure.

is reported that it achieves faster convergence in system mismatch, ${ }^{1}$ at the expense of slower convergence in echo return loss enhancement (ERLE), than other major preprocessing techniques such as in [5]. The scheme $e^{2}$ proposed in [23] utilizes the information from the two states of inputs simultaneously at each iteration. The two states can be associated with two states of solution sets (mathematically linear varieties [5]), say $\mathcal{V}$ and $\tilde{\mathcal{V}}$. By using the adaptive parallel subgradient projection (PSP) algorithm [28] (see Section 3.1), the scheme fairly reduces the zigzag $\operatorname{loss}^{3}$ shown in Figure 2(b), and the direction of its update is governed by certain weighting factors (see Figure 2(c)). However, the update direction realized by the uniform weights does not sufficiently approximate ideal one. Recently, an efficient strategic weight design called the pairwise optimal weight realization (POWER) was developed in $[31,32]$ for the adaptive PSP algorithm. The POWER technique realizes a best strategy (in the sense of pairwise and worst-case optimization) for the use of multiple information to determine the update direction. This suggests that further drastic acceleration is highly expected by exploiting POWER (see Figure 3).

In this paper, we propose a class of efficient fast SAEC schemes that further accelerate the method in [23] by employing POWER with keeping linear computational complexity. In fact, the POWER technique exerts far-reaching effects in a general adaptive filtering application, especially

\footnotetext{
${ }^{1}$ Recall that the fast and accurate estimation of $\mathbf{h}^{*}$ is necessary in SAEC, hence system mismatch is a very important criterion.

2 The scheme is derived from the adaptive projected subgradient method $[24,25]$, a unified framework for various adaptive filtering algorithms, which has also been applied to the multiple-access interference suppression problem in DS/CDMA systems successfully [26, 27].

${ }^{3}$ The loss is caused by the "small" angle between $\mathcal{V}$ and $\tilde{\mathcal{V}}$ due to the restriction of "slight" modification in preprocessing (see, e.g., [29, page 197] for angle between subspaces or linear varieties). Similar zigzag behavior can be observed for alternating projection methods known as Kaczmarz's method or, more generally, the projections onto convex sets (POCS) in convex feasibility problem; find a point in the nonempty intersection of fixed closed convex sets (see, e.g., [30] and Section 3.1). In the case of two subspaces $M_{1}$ and $M_{2}$, the rate of convergence of alternating projection methods is exactly given as $\left(\cos \left(M_{1}, M_{2}\right)\right)^{2 n-1}[29$, Theorem 9.31], where $\cos (\cdot, \cdot)$ denotes the cosine of the angle between two subspaces and $n$ the iteration number. This provides theoretical verification to slow convergence caused by the zigzag loss when the angle between two subspaces is small.
}

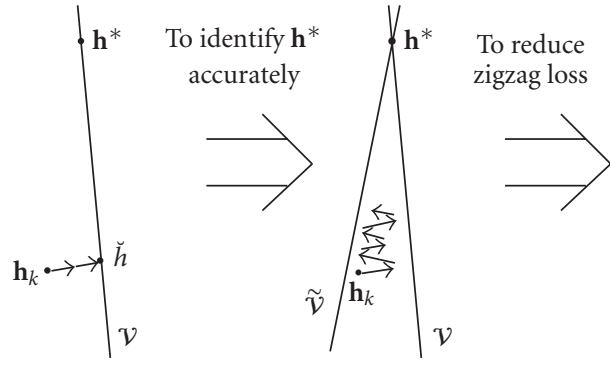

(a) Straightforward (b) Conventional

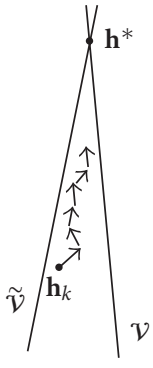

(c) UW-PSP
FIGURE 2: A geometric interpretation of existing methods: (a) straightforward: straightforward application of monaural scheme, (b) conventional: preprocessing-based approach with just one state of inputs at each iteration, (c) UW (uniform weight)-PSP: preprocessing-based approach with two state information at each iteration [23]. The solution set $\mathcal{V}$ is periodically changed into $\tilde{\mathcal{V}}$ by preprocessing ( $\mathcal{V}$ and $\tilde{\mathcal{V}}$ are linear varieties). Note that each arrow of "conventional" stands for the update accumulated during a halfcycle period in which the state of inputs is constant.

when the input signals are highly correlated. Hence, as seen from Figure 2, POWER is particularly suitable for the SAEC problem. The POWER technique is based on a simple formula to give the projection onto the intersection of two closed half-spaces ${ }^{4}$ that are defined by three vectors (see Proposition 1). We propose two schemes in the proposed class. The first scheme (Type I) exploits the formula in a combinatorial manner (see Figure 4(a)). The second scheme (Type II), on the other hand, exploits the formula just once after taking respective uniform averages of projections corresponding to each state of inputs (see Figure 4(b)). The latter scheme is computationally more efficient than the former one, while overall complexities, including the weight design, of both schemes are kept linear with respect to the filter length (see Remark 1(a)).

\footnotetext{
${ }^{4}$ Given $\mathbf{v} \in \mathscr{H}(\mathscr{H}:$ real Hilbert space $)$ and a closed subspace $M \subset \mathscr{H}$, the translation of $M$ by $\mathbf{v}$ defines the linear variety $V:=\mathbf{v}+M:=\{\mathbf{v}+\mathbf{m}: \mathbf{m} \in$ $M\}$. If $M^{\perp}:=\{\mathbf{x} \in \mathscr{H}:\langle\mathbf{x}, \mathbf{m}\rangle=0, \forall \mathbf{m} \in M\}$ satisfies $\operatorname{dim}\left(M^{\perp}\right)=1, V$ is called hyperplane, which can be expressed as $V=\{\mathbf{x} \in \mathscr{H}:\langle\mathbf{a}, \mathbf{x}\rangle=c\}$ for some $(\mathbf{0} \neq) \mathbf{a} \in \mathcal{H}$ and $c \in \mathbb{R} . \Pi^{-}:=\{\mathbf{x} \in \mathscr{H}:\langle\mathbf{a}, \mathbf{x}\rangle \leq c\}$ is called a closed half-space with its boundary $V$.
} 


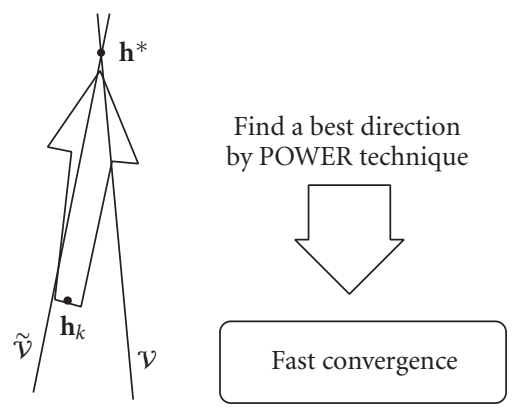

FIGURE 3: The direction of this paper.

Numerical examples demonstrate that notable improvements are achieved, in system mismatch as well as in ERLE, by the use of POWER in place of the uniform weights. Other possible ways to reduce the zigzag loss would be to employ the affine projection algorithm (APA) $[33,34]$ or the recursive least-squares (RLS) algorithm $[35,36]$ (the essential difference between our approach and APA is clearly described in Section 3.2). The proposed schemes are also compared with such other schemes, all of which employ the same preprocessing technique as the proposed schemes do. From our numerical experiments, we verify superiority of the proposed method. Moreover, we confirm that the proposed schemes exhibit excellent tracking behavior after a change of the echo paths.

\section{PRELIMINARIES}

\subsection{Stereo acoustic echo cancellation problem}

Throughout the paper, the following notations are used. Let $L \in \mathbb{N}^{*}:=\mathbb{N} \backslash\{0\}$ denote the length (of the impulse response) of the transmission path and $N \in \mathbb{N}^{*}$ the length of the echo path. For simplicity, let the length of the adaptive filter be $N$ (analyses for more general cases are presented in [5]). Referring to Figure 1, the signals at time $k \in \mathbb{N}$ are expressed as follows (the superscript $T$ stands for transposition):

(i) speech vector: $\boldsymbol{s}_{k} \in \mathbb{R}^{L}$;

(ii) ith transmission path: $\boldsymbol{\theta}_{(i)} \in \mathbb{R}^{L}(i=1,2)$;

(iii) $i$ th input: $u_{k}^{(i)}:=\mathbf{s}_{k}^{T} \boldsymbol{\theta}_{(i)} \in \mathbb{R}$;

(iv) ith input vector: $\mathbf{u}_{k}^{(i)}:=\left[u_{k}^{(i)}, u_{k-1}^{(i)}, \ldots, u_{k-N+1}^{(i)}\right]^{T} \in \mathbb{R}^{N}$;

(v) preprocessed version of $\mathbf{u}_{k}^{(1)}: \widetilde{\mathbf{u}}_{k}^{(1)} \in \mathbb{R}^{N}$;

(vi) input vector: $\mathbf{u}_{k}:=\left[\begin{array}{c}\widetilde{\mathbf{u}}_{k}^{(1)} \\ \mathbf{u}_{k}^{(2)}\end{array}\right] \in \mathscr{H}:=\mathbb{R}^{2 N}$;

(vii) input matrix: $\mathbf{U}_{k}:=\left[\mathbf{u}_{k}, \mathbf{u}_{k-1}, \ldots, \mathbf{u}_{k-r+1}\right] \in \mathbb{R}^{2 N \times r}$ $\left(r \in \mathbb{N}^{*}\right)$;

(viii) $i$ th echo path: $\mathbf{h}_{(i)}^{*} \in \mathbb{R}^{N}(i=1,2)$;

(ix) estimandum: $\mathbf{h}^{*}:=\left[\begin{array}{l}\mathbf{h}_{(1)}^{*} \\ \mathbf{h}_{(2)}^{*}\end{array}\right] \in \mathscr{H}$;

(x) adaptive filter (echo canceler): $\mathbf{h}_{k}:=\left[\begin{array}{l}\mathbf{h}_{k}^{(1)} \\ \mathbf{h}_{k}^{(2)}\end{array}\right] \in \mathscr{H}$;

(xi) noise: $\mathbf{n}_{k}:=\left[n_{k}, n_{k-1}, \ldots, n_{k-r+1}\right]^{T} \in \mathbb{R}^{r}$;

(xii) output: $\mathbf{d}_{k}:=\mathbf{U}_{k}^{T} \mathbf{h}^{*}+\mathbf{n}_{k} \in \mathbb{R}^{r}$;

(xiii) residual error function: $\mathbf{e}_{k}(\mathbf{h}):=\mathbf{U}_{k}^{T} \mathbf{h}-\mathbf{d}_{k} \in \mathbb{R}^{r}$.
Current state 0th stage 1st stage 2nd stage Final stage

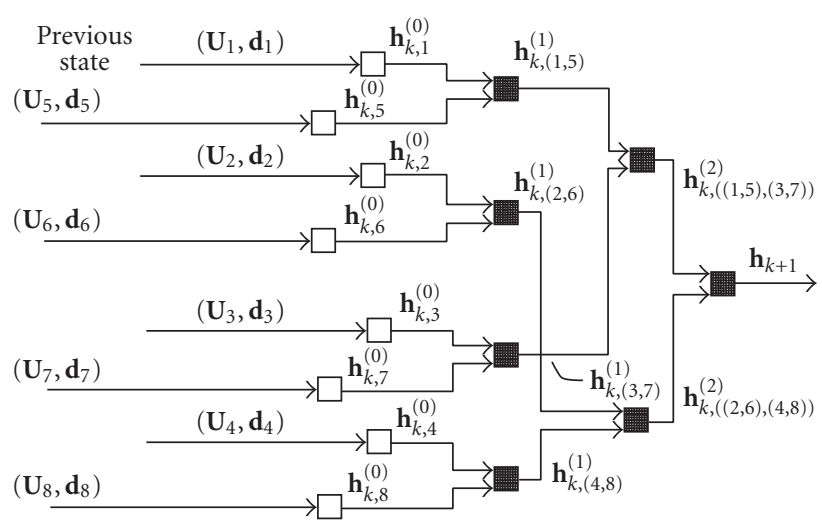

Projection

POWER

(a)

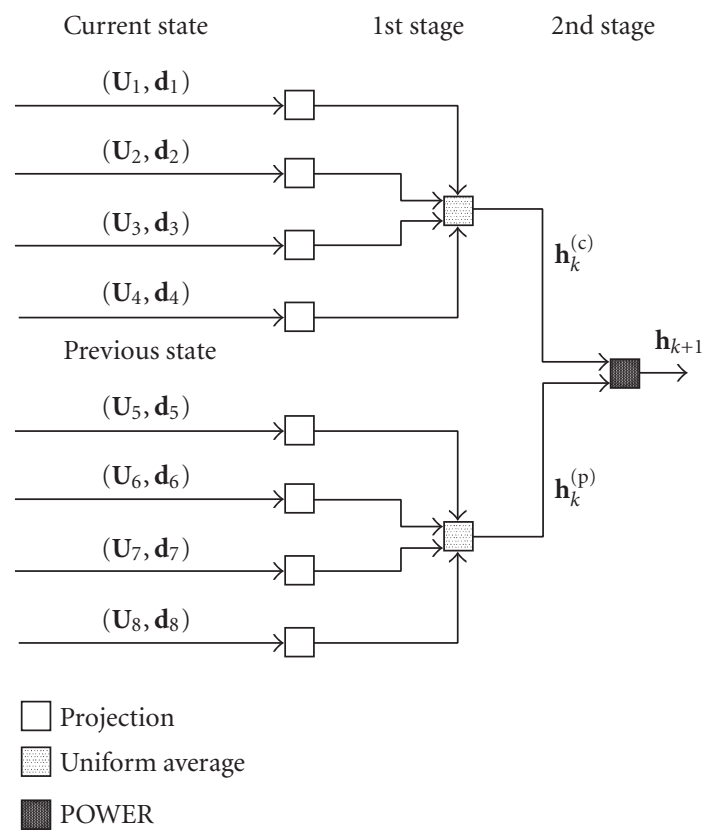

(b)

FIGURE 4: Simple system models with eight parallel processors $(q=$ 4) to implement (a) POWER I and (b) POWER II. For notational simplicity, define the current control sequence $\ell_{k}^{(\mathrm{c})}=\{1,2,3,4\}$ and the previous control sequence $l_{k}^{(\mathrm{p})}=\{5,6,7,8\}$. This type of design of control sequences for POWER I is called binary-tree-like construction. It is seen that POWER II is more efficient in computation than POWER I.

Here, $\mathscr{H}\left(:=\mathbb{R}^{2 N}\right)$ is a real Hilbert space equipped with the inner product $\langle\mathbf{x}, \mathbf{y}\rangle:=\mathbf{x}^{T} \mathbf{y}, \forall \mathbf{x}, \mathbf{y} \in \mathcal{H}$, and its induced norm $\|\mathbf{x}\|:=\left(\mathbf{x}^{T} \mathbf{x}\right)^{1 / 2}, \forall \mathbf{x} \in \mathcal{H}$. For any nonempty closed convex set $C \subset \mathscr{H}$, the projection operator $P_{C}: \mathscr{H} \rightarrow C$ is defined by $\left\|\mathbf{x}-P_{C}(\mathbf{x})\right\|=\min _{\mathbf{y} \in C}\|\mathbf{x}-\mathbf{y}\|, \forall \mathbf{x} \in \mathscr{H}$. The notation $|S|$ stands for the cardinality of a set $S$. 
The goal of the SAEC problem is to cancel the echo stably, that is, $\mathbf{u}_{k}^{T} \mathbf{h}^{*}-\mathbf{u}_{k}^{T} \mathbf{h}_{k} \approx 0$, for all $k \in \mathbb{N}$. Since only $\mathbf{u}_{k}$ and $\mathbf{d}_{k}$ are observable, a common alternative goal is to suppress the residual echo; that is, $\mathbf{e}_{k}\left(\mathbf{h}_{k}\right) \approx \mathbf{0}$, for all $k \in \mathbb{N}$.

\subsection{Nonuniqueness problem}

In 1991, Sondhi and Morgan found unacceptably slow convergence phenomena in SAEC [2] and, in 1995, Sondhi et al. showed that the primitive solution set, obtained from the normal equation to be solved for minimization of the residual echo, is too large and it depends on the transmission paths (due to inherent dependency caused by highly crosscorrelated stereo input signals) [3]. This fundamental difficulty, deeply seated in SAEC, is commonly referred to as the nonuniqueness problem, which has earned recognition as an intrinsic burden not existing in the monaural echo cancellation. In 1998, Benesty et al. further clarified this problem, and showed that the normal equation is often ill-conditioned or has infinitely many solutions [5].

Let us simply explain the nonuniqueness problem mathematically. The input sequence $\left(u_{k}^{(i)}\right)_{k \in \mathbb{N}}, i=1,2$, can be written as

$$
u_{k}^{(i)}=s_{k} * \boldsymbol{\theta}_{(i)}
$$

where $*$ denotes convolution. Considering the case of $N=L$, for simplicity,

$$
\breve{\mathbf{h}}:=\left[\begin{array}{c}
\breve{\mathbf{h}}^{(1)} \\
\breve{\mathbf{h}}^{(2)}
\end{array}\right]:=\mathbf{h}^{*}+\alpha\left[\begin{array}{c}
\boldsymbol{\theta}_{(2)} \\
-\boldsymbol{\theta}_{(1)}
\end{array}\right], \quad \alpha \in \mathbb{R},
$$

satisfies

$$
\sum_{i=1,2} u_{k}^{(i)} * \breve{\mathbf{h}}^{(i)}=\sum_{i=1,2} u_{k}^{(i)} * \mathbf{h}_{(i)}^{*},
$$

which implies, under noiseless environments, that $\mathbf{e}_{k}(\breve{\mathbf{h}})=\mathbf{0}$. This is the basic mechanism of the nonuniqueness problem [5] (precise analysis is possible by using $z$-transform of (3) with (1); see, e.g., [10]). From (2), we see that filter coefficients that cancel the echo depend on the transmission paths $\boldsymbol{\theta}_{(1)}$ and $\boldsymbol{\theta}_{(2)}$. This implies that, without well-approximating $\mathbf{h}^{*}$, echo will relapse by change of $\boldsymbol{\theta}_{(1)}$ and $\boldsymbol{\theta}_{(2)}$ due to talker's alternation, and so forth (see also [23, Appendix A]). Hence, it is strongly desired to keep $\mathbf{h}_{k}$ close to $\mathbf{h}^{*}$ before the transmission paths change drastically.

\section{PROPOSED CLASS OF STEREO ACOUSTIC ECHO CANCELLATION SCHEMES}

In this section, we present a class of set-theoretic SAEC schemes based on the POWER weighting technique. The proposed approach utilizes parallel projection onto certain closed convex sets. First, we provide a brief introduction of set-theoretic adaptive filtering and define the closed convex sets. Then, we show the relationship between the proposed approach and the APA-based method. Finally, we present the proposed schemes in a simple manner.

\subsection{Set-theoretic adaptive filtering and convex set design}

We briefly introduce the basic idea of the set-theoretic [24, $25,28,37,38] /$ set-membership $[39,40]$ approaches in the adaptive filtering. Let us first start with the set-theoretic approach $^{5}$ in the static convex feasibility problem [30, 37, 38, 41]; find a point in the nonempty intersection of fixed closed convex sets $S_{i}, i \in \ell \subset \mathbb{N}$. Each set $S_{i}$ is designed based on available information, such as noise statistics and observed data, so that $S_{i}$ contains the estimandum $\mathbf{h}^{*}$ with high probability. Suppose that $\mathbf{h}^{*} \in S_{i}$, for all $i \in \ell$. Then, it is a natural strategy to find a point in $\bigcap_{i \in \ell} S_{i}$ as an estimate of $\mathbf{h}^{*}$. Due to the nonlinear nature of the problem, certain successive numerical approximations by utilizing the information on each set $S_{i}$ infinitely many times are, in general, necessary.

In [28], the adaptive filtering problem is translated into a time-varying version of the convex feasibility problem, where multiple closed convex sets $S_{i}^{(k)}, i \in \ell_{k} \subset \mathbb{N}$, are defined by multiple observable data, hence being time-varying (a unified framework for this approach is found in $[24,25])$. Namely, the collection of convex sets $\left(S_{i}^{(k)}\right)_{i \in \ell_{k}}$ used at time $k$ is varying based on data incoming from one minute to the next (also $\mathbf{h}^{*}$ is possibly time-varying). Especially in rapidly time-varying environments, it should be reasonable to use a limited number of sets $\left(S_{i}^{(k)}\right)_{i \in \ell_{k}}$ that are defined with recently obtained data. This strategy agrees with saving the computational complexity, another requirement in adaptive filtering. This is the basic idea of the set-theoretic adaptive filtering approach.

The adaptive PSP algorithm [28] was proposed as an efficient set-theoretic adaptive filtering technique. The algorithm adopts subgradient projections as approximations of the exact projections onto the convex sets for saving the computation costs. The multiple (subgradient) projections are computed in parallel, hence the algorithm can save, by engaging parallel processors, the time consumption for each update. Finally, the update direction of filter is determined by taking a weighted average of the projections.

The first step is to define closed convex sets that contain $\mathbf{h}^{*}$ with high probability. A possible choice is as follows [28]:

$$
\begin{array}{r}
C_{l}(\rho):=\left\{\mathbf{h} \in \mathcal{H}\left(:=\mathbb{R}^{2 N}\right): g_{\iota}(\mathbf{h}):=\left\|\mathbf{e}_{\iota}(\mathbf{h})\right\|^{2}-\rho \leq 0\right\} \\
\forall \iota \in \ell_{k} \subset \mathbb{N}, \quad \forall k \in \mathbb{N},
\end{array}
$$

where $\rho \geq 0$ and $\ell_{k}$ is the control sequence at time $k$ (see Section 3.3). Assignment of an appropriate value to $\rho$ raises the membership probability $\operatorname{Prob}\left\{\mathbf{h}^{*} \in C_{l}(\rho)\right\}$ and, at the same time, keeps $C_{l}(\rho)$ sufficiently small (see Section 3.2 for detailed discussion). Since the projection onto $C_{l}(\rho)$ requires, in general, very high computational complexity, we

\footnotetext{
5 The difference is clearly stated in [37] between the set-theoretic approach and the conventional approach, that is, optimize an objective function with or without constraints.
} 
instead employ the projection onto the closed half-space ${ }^{6}$ $[28] H_{\imath}^{-}\left(\mathbf{h}_{k}\right):=\left\{\mathbf{x} \in \mathscr{H}:\left\langle\mathbf{x}-\mathbf{h}_{k}, \nabla g_{\iota}\left(\mathbf{h}_{k}\right)\right\rangle+g_{\iota}\left(\mathbf{h}_{k}\right) \leq 0\right\} \supset$ $C_{l}(\rho)$, which has the following simple closed-form expression:

$$
\begin{aligned}
& P_{H_{l}^{-}\left(\mathbf{h}_{k}\right)}(\mathbf{h}) \\
& = \begin{cases}\mathbf{h}+\frac{-g_{\iota}\left(\mathbf{h}_{k}\right)+\left(\mathbf{h}_{k}-\mathbf{h}\right)^{T} \nabla g_{\iota}\left(\mathbf{h}_{k}\right)}{\left\|\nabla g_{\iota}\left(\mathbf{h}_{k}\right)\right\|^{2}} \nabla g_{\iota}\left(\mathbf{h}_{k}\right) & \text { if } \mathbf{h} \notin H_{\iota}^{-}\left(\mathbf{h}_{k}\right), \\
\mathbf{h} & \text { otherwise. }\end{cases}
\end{aligned}
$$

Here, $\nabla g_{l}\left(\mathbf{h}_{k}\right)=2 \mathbf{U}_{\iota} \mathbf{e}_{l}\left(\mathbf{h}_{k}\right)$ and $P_{H_{l}^{-}\left(\mathbf{h}_{k}\right)}(\mathbf{h}) \cong P_{C_{l}(\rho)}(\mathbf{h})$; see [28, Figure 3]. It should be remarked that $P_{H_{l}^{-}\left(\mathbf{h}_{k}\right)}(\mathbf{h})$ requires $O(N)$ complexity. Choosing specially $\mathbf{h}=\mathbf{h}_{k}$, we have

$$
\begin{aligned}
& P_{H_{l}^{-}\left(\mathbf{h}_{k}\right)}\left(\mathbf{h}_{k}\right) \\
& \quad= \begin{cases}\mathbf{h}_{k}-\frac{g_{\iota}\left(\mathbf{h}_{k}\right)}{\left\|\nabla g_{\iota}\left(\mathbf{h}_{k}\right)\right\|^{2}} \nabla g_{\iota}\left(\mathbf{h}_{k}\right) & \text { if } \mathbf{h}_{k} \notin H_{\iota}^{-}\left(\mathbf{h}_{k}\right), \\
\mathbf{h}_{k} & \text { otherwise. }\end{cases}
\end{aligned}
$$

\subsection{Relationship to APA-based method and robustness issue against noise}

The popular APA [34] can be viewed in the time-varying set-theoretic framework [28] with the linear varieties $V_{k}:=$ $\arg \min _{\mathbf{h} \in \mathscr{H}}\left\|\mathbf{e}_{k}(\mathbf{h})\right\|^{2}(\forall k \in \mathbb{N})$. The APA generates a sequence of filtering vectors $\left(\mathbf{h}_{k}\right)_{k \in \mathbb{N}} \subset \mathcal{H}\left(:=\mathbb{R}^{2 N}\right)$ by (see [28])

$$
\mathbf{h}_{k+1}=\mathbf{h}_{k}+\lambda_{k}\left(P_{V_{k}}\left(\mathbf{h}_{k}\right)-\mathbf{h}_{k}\right), \quad \forall k \in \mathbb{N}
$$

where $\lambda_{k} \in(0,2)$. In particular, for $r=1,(7)$ is nothing but the normalized least-mean-square (NLMS) algorithm [43], where $r$ is the dimension of affine projection (see Section 2.1 for the definitions of $\mathbf{U}_{k} \in \mathbb{R}^{2 N \times r}$ and $\mathbf{d}_{k} \in \mathbb{R}^{r}$ ). A simple comparison of $V_{k}$ with $C_{k}(\rho)$ in (4) implies that $V_{k}=C_{k}\left(\delta_{k}\right)$, where $\delta_{k}:=\min _{\mathbf{h} \in \mathscr{H}}\left\|\mathbf{e}_{k}(\mathbf{h})\right\|^{2}$. Note here that we most likely have $\delta_{k} \approx 0$, since we often have $2 N \gg r$ due to long impulse responses of acoustic paths.

The remains of this section is devoted to the robustness issue against noise by highlighting the membership $\mathbf{h}^{*} \in$ $C_{k}(\rho)$, which ensures the monotone approximation property (for stability), that is, $\left\|\mathbf{h}_{k+1}-\mathbf{h}^{*}\right\| \leq\left\|\mathbf{h}_{k}-\mathbf{h}^{*}\right\|$. Noting that $\mathbf{h}^{*} \in C_{k}(\rho) \Leftrightarrow\left\|\mathbf{e}_{k}\left(\mathbf{h}^{*}\right)\right\|^{2}=\left\|\mathbf{n}_{k}\right\|^{2} \leq \rho$, we see that $\rho$ governs the reliability on the membership $\mathbf{h}^{*} \in C_{k}(\rho)$ by $\int_{0}^{\rho} f_{r}(\xi) d \xi$, where $f_{r}(\xi)$ is the probability density function (pdf) of the random variable $\xi:=\left\|\mathbf{n}_{k}\right\|^{2},\left(f_{r}(\xi)\right.$ is given in [28, Equation (9)]). Under the assumption that the noise process is a zeromean i.i.d. Gaussian random variable $\mathcal{N}\left(0, \sigma^{2}\right)$, the random variable $\xi$ follows a $\chi^{2}$ distribution (of order $r$ ), where $\sigma^{2}$ is

\footnotetext{
6 Tighter closed half-spaces are also presented in [42], which can also be used with the proposed schemes.
}

the variance of noise. The pdf $f_{r}(\xi)$ is strictly monotone decreasing over $\xi \geq 0$ for $r=1,2$, whereas for $r \geq 3$, it has its unique peak at $\xi=(r-2) \sigma^{2}$ and $f_{r}(0)=\lim _{\xi \rightarrow \infty} f_{r}(\xi)=0$. Recall that we most likely have $\delta_{k} \approx 0$. The above facts imply that for $r \geq 3, \operatorname{Prob}\left\{\mathbf{h}^{*} \in C_{k}\left(\delta_{k}\right)\left(=V_{k}\right)\right\}$ is expected to be small, which causes serious sensitivity of the APA to noise for $r \geq 3$ (see Section 4). For $r=1,2$, on the other hand, Prob $\left\{\mathbf{h}^{*} \in C_{k}\left(\delta_{k}\right)\right\}$ is expected to be relatively large, which suggests robustness of the APA $(r=1,2)$ against noise (this agrees with the $H^{\infty}$ optimality [44] of the NLMS, a special case of the APA for $r=1$ ). By designing appropriate $\rho$ based on statistics of noise process (see [28, Example 1]), the proposed schemes can fairly raise $\operatorname{Prob}\left\{\mathbf{h}^{*} \in C_{k}(\rho)\right\}$; note that $\operatorname{Prob}\left\{\mathbf{h}^{*} \in H_{k}^{-}\left(\mathbf{h}_{k}\right)\right\} \geq \operatorname{Prob}\left\{\mathbf{h}^{*} \in C_{k}(\rho)\right\}$ because $H_{k}^{-}\left(\mathbf{h}_{k}\right) \supset C_{k}(\rho)$. This brings about the noise robustness of POWER I/II in Section 3.3.

\subsection{Novel POWER-based stereo echo canceler}

Given $q \in \mathbb{N}^{*}$, define the control sequence consisting of the $q$ latest time indices as $\ell_{k}^{(\mathrm{c})}:=\{k, k-1, \ldots, k-q+1\} \subset \mathbb{N}$. Let $Q \in \mathbb{N}^{*}$ denote the cycle period of preprocessing $[14,15]$, that is, every $Q / 2$ iterations, the state of inputs is switched. Then, $k-Q / 2(\forall k>Q / 2)$ always belongs to the state opposite to $k$. To utilize data from both states of inputs, we use $\ell_{k}^{(\mathrm{c})} \cup \ell_{k}^{(\mathrm{p})}$ as in [23], where

$$
l_{k}^{(\mathrm{p})}:= \begin{cases}\varnothing, & 0 \leq k \leq \frac{Q}{2}, \\ l_{k-\mathrm{Q} / 2}^{(\mathrm{c})}, & k>\frac{Q}{2} .\end{cases}
$$

Note that the definitions of $\ell_{k}^{(\mathrm{c})}$ and $\ell_{k}^{(\mathrm{p})}$ can be generalized to any index sets consisting of arbitrary indices chosen from the current and previous states, respectively (see [45]). For simplicity, however, we focus on the above specific definition in the following.

The most important definition is now given: three-point expression of projection onto the intersection of two closed half-spaces. For convenience, let us define that for all $\mathbf{a}, \mathbf{b} \in$ $\mathscr{H}$,

$$
\Pi^{-}(\mathbf{a}, \mathbf{b}):=\{\mathbf{y} \in \mathscr{H}:\langle\mathbf{a}-\mathbf{b}, \mathbf{y}-\mathbf{b}\rangle \leq 0\} \subset \mathcal{H},
$$

where $\Pi^{-}(\mathbf{a}, \mathbf{b})$ is a closed half-space if $\mathbf{a} \neq \mathbf{b}$. Then, for a given ordered triplet $(\mathbf{s}, \mathbf{a}, \mathbf{b}) \in \mathcal{H}^{3}$ such that $\Pi^{-}(\mathbf{s}, \mathbf{a}) \cap$ $\Pi^{-}(\mathbf{s}, \mathbf{b}) \neq \varnothing$, we define

$$
\mathcal{P}(\mathbf{s}, \mathbf{a}, \mathbf{b}):=P_{\Pi^{-}(\mathbf{s}, \mathbf{a}) \cap \Pi^{-}(\mathbf{s}, \mathbf{b})}(\mathbf{s}),
$$

namely $\mathcal{P}(\mathbf{s}, \mathbf{a}, \mathbf{b})$ denotes the projection of $\mathbf{s}$ onto $\Pi^{-}(\mathbf{s}, \mathbf{a}) \cap$ $\Pi^{-}(\mathbf{s}, \mathbf{b})$ in $\mathcal{H}$. How to compute $\mathcal{P}(\mathbf{s}, \mathbf{a}, \mathbf{b})$ is given in Appendix C. 
We propose a new class of SAEC schemes that utilize $\mathcal{P}(\mathbf{s}$, a,b) (Proposition 1) to realize better weights in the method proposed in [23] (see Appendix B). Two schemes in the proposed class are presented below, where two families of closed half-spaces, $\left\{H_{\iota}^{-}\left(\mathbf{h}_{k}\right)\right\}_{\iota \in \ell_{k}^{(c)}}$ and $\left\{H_{l}^{-}\left(\mathbf{h}_{k}\right)\right\}_{l \in \ell_{k}^{(\mathrm{p})}}$, are used in different ways.

\subsubsection{POWER TypeI}

A scheme that exploits the POWER technique in a combinatorial manner is presented below (see Figure 4(a)). Define $l_{k}^{(1)}:=\{(k-i+1, k-Q / 2-i+1): i=1,2, \ldots, q\} \subset$ $\left\{\left(\iota_{1}, \iota_{2}\right): \iota_{1} \in l_{k}^{(\mathrm{c})}, \iota_{2} \in l_{k}^{(\mathrm{p})}\right\}$. Also define inductively the control sequences used in each stage as $\ell_{k}^{(m)} \subset\left\{\left(\iota_{1}, \iota_{2}\right)\right.$ : $\left.\iota_{1}, \iota_{2} \in \ell_{k}^{(m-1)}, \iota_{1} \neq \iota_{2}\right\}, \forall m \in\{2,3, \ldots, M\}$, for all $k \in \mathbb{N}$, satisfying $1=\left|\boldsymbol{l}_{k}^{(M)}\right| \subsetneq\left|\boldsymbol{\ell}_{k}^{(M-1)}\right| \leq \cdots \leq\left|\boldsymbol{\ell}_{k}^{(2)}\right| \leq\left|\boldsymbol{\ell}_{k}^{(1)}\right|=q$. The scheme is given as follows.

Scheme 1 (POWER Type I). Suppose that a sequence of closed convex sets $\left(C_{k}(\rho)\right)_{k \in \mathbb{N}} \subset \mathscr{H}$ is defined as in (4). Let $\mathbf{h}_{0} \in \mathscr{H}$ be an arbitrarily chosen initial vector. Then, define a sequence of filtering vectors $\left(\mathbf{h}_{k}\right)_{k \in \mathbb{N}} \subset \mathscr{H}$ through multiple stages.

\section{Oth stage: projection onto $2 q$ half-spaces}

$$
\mathbf{h}_{k, l}^{(0)}:=P_{H_{l}^{-}\left(\mathbf{h}_{k}\right)}\left(\mathbf{h}_{k}\right), \quad \forall k \in \mathbb{N}, \forall \iota \in l_{k}^{(\mathrm{c})} \cup \ell_{k}^{(\mathrm{p})},
$$

where $P_{H_{l}^{-}\left(\mathbf{h}_{k}\right)}\left(\mathbf{h}_{k}\right)$ is computed by (6).

\section{$1 s t \sim$ Mth stage: find good direction}

for $m:=1$ to $M$ do

$$
\begin{aligned}
\mathbf{h}_{k, l}^{(m)}:= \begin{cases}\mathbf{h}_{k} & \text { if } \eta_{k, l}^{(m)}=-\sqrt{\xi_{k, l}^{(m)} \zeta_{k, l}^{(m)}} \neq 0, \\
\mathcal{P}\left(\mathbf{h}_{k}, \mathbf{h}_{k, l_{1}}^{(m-1)}, \mathbf{h}_{k, l_{2}}^{(m-1)}\right) & \text { otherwise, }\end{cases} \\
\forall k \in \mathbb{N}, \quad \forall \iota=\left(\iota_{1}, \iota_{2}\right) \in \boldsymbol{l}_{k}^{(m)},
\end{aligned}
$$

where $\eta_{k, l}^{(m)}:=\left\langle\mathbf{h}_{k, l_{1}}^{(m-1)}-\mathbf{h}_{k}, \mathbf{h}_{k, l_{2}}^{(m-1)}-\mathbf{h}_{k}\right\rangle, \xi_{k, l}^{(m)}:=\| \mathbf{h}_{k, l_{1}}^{(m-1)}-$ $\mathbf{h}_{k} \|^{2}$, and $\zeta_{k, l}^{(m)}:=\left\|\mathbf{h}_{k, l_{2}}^{(m-1)}-\mathbf{h}_{k}\right\|^{2}$.

end.

\section{Final stage: update to good direction}

$$
\mathbf{h}_{k+1}:=\mathbf{h}_{k}+\lambda_{k}\left(\mathbf{h}_{k, l}^{(M)}-\mathbf{h}_{k}\right), \quad \forall k \in \mathbb{N}
$$

where $\lambda_{k} \in[0,2]$ is the step size.
Through the multiple stages, the direction of update is improved thanks to the operator $\mathcal{P}(\cdot, \cdot, \cdot)$ (see [32] for details).

\subsubsection{POWER Type II}

A simple and efficient scheme that exploits the POWER technique just once is given as follows (see Figure 4(b)).

Scheme 2 (POWER Type II). Suppose that a sequence of closed convex sets $\left(C_{l}(\rho)\right)_{\iota \in \ell} \subset \mathscr{H}$ is defined as in (4), where $\ell:=\bigcup_{k \in \mathbb{N}}\left(l_{k}^{(\mathrm{c})} \cup \ell_{k}^{(\mathrm{p})}\right)$. Let $\mathbf{h}_{0} \in \mathcal{H}$ be an arbitrarily chosen initial vector. Then, define a sequence of filtering vectors $\left(\mathbf{h}_{k}\right)_{k \in \mathbb{N}} \subset \mathscr{H}$ through the following two stages.

\section{1st stage: uniformly averaged directions}

$$
\begin{aligned}
& \mathbf{h}_{k}^{(\mathrm{g})} \\
& := \begin{cases}\mathbf{h}_{k}+\mathcal{M}_{k}^{(\mathrm{g})}\left(\sum_{i \in l_{k}^{(\mathrm{g})}} w_{k}^{(\mathrm{g})} P_{H_{l}^{-}\left(\mathbf{h}_{k}\right)}\left(\mathbf{h}_{k}\right)-\mathbf{h}_{k}\right) & \text { if } \boldsymbol{l}_{k}^{(\mathrm{g})} \neq \varnothing, \\
\mathbf{h}_{k} & \text { otherwise, } \\
& \forall k \in \mathbb{N}, \quad \forall \mathrm{g} \in\{\mathrm{c}, \mathrm{p}\},\end{cases}
\end{aligned}
$$

where $w_{k}^{(\mathrm{g})}:=1 /\left|\boldsymbol{l}_{k}^{(\mathrm{g})}\right|=1 / q\left(\forall \iota \in \ell_{k}^{(\mathrm{g})}\right)$ and

$$
\begin{aligned}
& \mathcal{M}_{k}^{(\mathrm{g})} \\
& := \begin{cases}\frac{\sum_{\iota \in l_{k}^{(\mathrm{g})}} w_{k}^{(\mathrm{g})}\left\|P_{H_{l}^{-}\left(\mathbf{h}_{k}\right)}\left(\mathbf{h}_{k}\right)-\mathbf{h}_{k}\right\|^{2}}{\left\|\sum_{l \in l_{k}^{(\mathrm{g})}} w_{k}^{(\mathrm{g})} P_{H_{l}^{-}\left(\mathbf{h}_{k}\right)}\left(\mathbf{h}_{k}\right)-\mathbf{h}_{k}\right\|^{2}} & \text { if } \mathbf{h}_{k} \notin \bigcap_{l \in l_{k}^{(\mathrm{g})} H_{l}^{-}}\left(\mathbf{h}_{k}\right), \\
1 & \text { otherwise. }\end{cases}
\end{aligned}
$$

\section{2nd stage: reasonably averaged direction by POWER}

$$
\mathbf{h}_{k+1}:= \begin{cases}\mathbf{h}_{k} & \text { if } \eta_{k}=-\sqrt{\xi_{k} \zeta_{k}} \neq 0, \\ \mathbf{h}_{k}+\lambda_{k}\left\{\mathcal{P}\left(\mathbf{h}_{k}, \mathbf{h}_{k}^{(\mathrm{c})}, \mathbf{h}_{k}^{(\mathrm{p})}\right)-\mathbf{h}_{k}\right\} & \text { otherwise, }\end{cases}
$$

for all $k \in \mathbb{N}$, where $\lambda_{k} \in[0,2]$ is the step size, $\eta_{k}:=\left\langle\mathbf{h}_{k}^{(\mathrm{c})}-\right.$ $\left.\mathbf{h}_{k}, \mathbf{h}_{k}^{(\mathrm{p})}-\mathbf{h}_{k}\right\rangle, \xi_{k}:=\left\|\mathbf{h}_{k}^{(\mathrm{c})}-\mathbf{h}_{k}\right\|^{2}$, and $\zeta_{k}:=\left\|\mathbf{h}_{k}^{(\mathrm{p})}-\mathbf{h}_{k}\right\|^{2}$.

In the 1st stage, for saving the computational complexity, the uniform averages $\mathbf{h}_{k}^{(\mathrm{c})}$ and $\mathbf{h}_{k}^{(\mathrm{p})}$ are computed for two groups corresponding to $\ell_{k}^{(\mathrm{c})}$ and $\ell_{k}^{(\mathrm{p})}$. In the 2 nd stage, the POWER technique is exploited to find a good direction of update based on three kinds of information: $\mathbf{h}_{k}, \mathbf{h}_{k}^{(\mathrm{c})}$, and $\mathbf{h}_{k}^{(\mathrm{p})}$ (see [32] for details). 


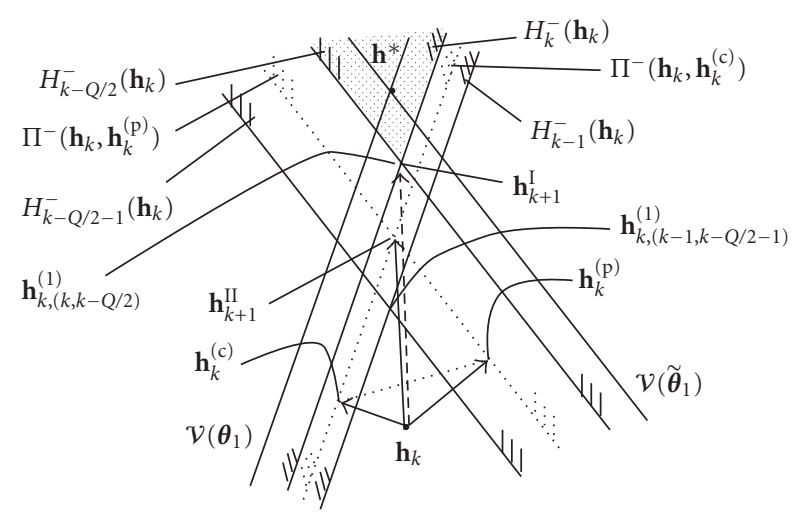

FIGURE 5: A geometric interpretation of the proposed schemes. POWER I: $\mathbf{h}_{k+1}^{\mathrm{I}}$, POWER II: $\mathbf{h}_{k+1}^{\mathrm{II}}$. The control sequences are defined as $\ell_{k}^{(\mathrm{c})}=\{k, k-1\}$ and $\ell_{k}^{(\mathrm{p})}=\{k-Q / 2, k-Q / 2-1\}$. The dotted area shows $\bigcap_{\iota \in \ell_{k}^{(\mathrm{c})} \cup \ell_{k}^{(\mathrm{p})}} H_{l}^{-}\left(\mathbf{h}_{k}\right)$.

Remark 1. (a) Simple system models to implement the proposed schemes with $q=4$ are shown in Figure 4 . The structure of POWER I is named binary-tree-like construction with its number of stages $M=\left\lceil\log _{2} q\right\rceil+1$; in this case, $M=3$ (see $[31,32]$ ). We see that POWER II is more efficient in computational complexity than POWER I, since it utilizes the POWER technique just once. The projections $\left\{P_{H_{l}^{-}\left(\mathbf{h}_{k}\right)}\left(\mathbf{h}_{k}\right)\right\}_{l \in \ell_{k}^{(\mathrm{c})} \cup \ell_{k}^{(\mathrm{p})}}$, for all $k \in \mathbb{N}$, in (11) and (14) are, respectively, computed simultaneously with $2 q$ concurrent processors. This implies that the proposed schemes are inherently suitable for implementation with concurrent processors. With such processors, the number of multiplications imposed on each processor is $(3 M+2 r+1) N+21 M+r$ $\left(M=\left\lceil\log _{2} q\right\rceil+1\right)$ for POWER I and $(2 r+6) N+r$ for POWER II for $q \geq 2$; for $q=1$, it is reduced to $(2 r+4) N+r$ for POWER I/II (see [32]). In other words, the complexity is kept $O(N)$, which is a desired property especially for realtime implementation.

(b) Discussions about convergence of the adaptive PSP algorithm are found in the adaptive projected subgradient method [24, 25], a more general framework. A geometric interpretation illustrated in Figure 5 will be rather helpful from a standpoint of application. For simplicity, we set $q=2$ and $\lambda_{k}=1$. In the figure, the estimandum $\mathbf{h}^{*}$ (see Section 2.1) is assumed to belong to the dotted area, that is, $\mathbf{h}^{*} \in \bigcap_{l \in \ell_{k}^{(\mathrm{c})} \cup \ell_{k}^{(\mathrm{p})}} H_{l}^{-}\left(\mathbf{h}_{k}\right)$. This assumption holds if $C_{k}(\rho)$ is defined appropriately (for details, see [28]). We see that the schemes realize good directions of update. For visual clarity, the half-spaces $\Pi^{-}\left(\mathbf{h}_{k}, \mathbf{h}_{k,(k, k-Q / 2)}^{(1)}\right)$ and $\Pi^{-}\left(\mathbf{h}_{k}, \mathbf{h}_{k,(k-1, k-Q / 2-1)}^{(1)}\right)$ are omitted. It is not hard to see that $\mathbf{h}_{k+1}=\mathcal{P}\left(\mathbf{h}_{k}, \mathbf{h}_{k,(k, k-Q / 2)}^{(1)}, \mathbf{h}_{k,(k-1, k-Q / 2-1)}^{(1)}\right)=\mathbf{h}_{k,(k, k-Q / 2)}^{(1)}$ in this simple example.

(c) The proposed schemes realize strategic weight designs for the method in [23] in the sense that the schemes give optimal weights, based on a certain max-min criterion, in each stage, see Appendices C and D.

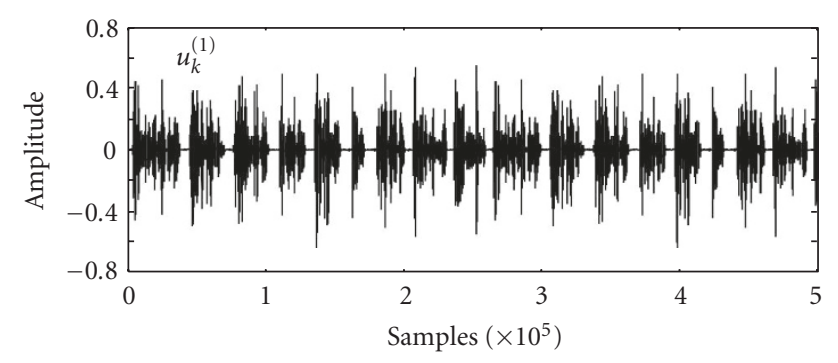

(a)

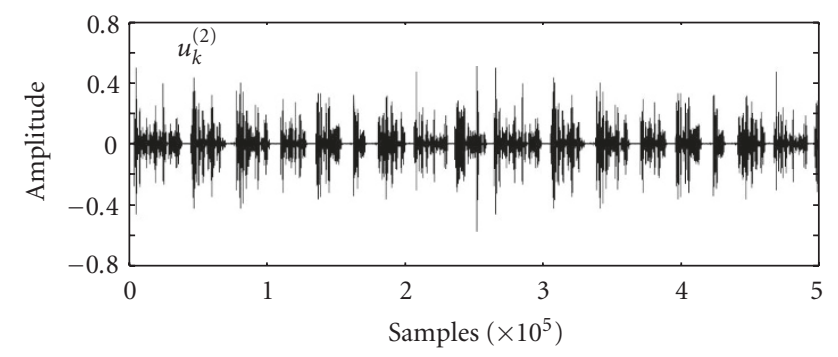

(b)

FIgURE 6: The input signals $\left(u_{k}^{(1)}\right)_{k \in \mathbb{N}}$ and $\left(u_{k}^{(2)}\right)_{k \in \mathbb{N}}$. The signals are generated from a speech signal, sampled at $8 \mathrm{kHz}$, of an English native male.

\section{NUMERICAL EXAMPLES}

This section presents numerical examples of the proposed schemes, the UW-PSP [23] (see Appendix B), APA [33, 34], NLMS [43], and fast RLS (FRLS) [36, 46] algorithms. All the methods are performed with a common preprocessing technique in $[14,15]$ that periodically delays input signals in the 1st channel with the cycle of preprocessing $Q=$ 2000. The tests are conducted, for estimating $\mathbf{h}^{*} \in \mathcal{H}:=$ $\mathbb{R}^{2000}(N=L=1000)$, under the noise situation of SNR := $10 \log _{10}\left(E\left\{z_{k}^{2}\right\} / E\left\{n_{k}^{2}\right\}\right)=25 \mathrm{~dB}$, where $z_{k}:=\left\langle\mathbf{u}_{k}, \mathbf{h}^{*}\right\rangle$ and $E\{\cdot\}$ denote pure echo (i.e., echo without noise) and expectation, respectively. We utilize a recorded speech signal of an English native male ${ }^{7}$ shown in Figure 6, for $\left(s_{k}\right)_{k \in \mathbb{N}}$, which was sampled at $8 \mathrm{kHz}$. For numerical stability against the poorly excited inputs observed in Figure 6, all the algorithms are regularized. The APA is regularized by following the way in [47] with exactly the same parameter as in [28]. The NLMS is regularized by following the way in [35, Equation (9.144)] with the regularization parameter $\delta=1.0 \times 10^{-1}$ for better performance. Because the original RLS algorithm is computationally intensive for acoustic echo cancellation applications [11, page 77], a simplified implementation of the regularized RLS [46] is employed with $\xi_{k}^{2}=20 \sigma_{u}^{2}$ and $\phi_{k}=1(\forall k \in \mathbb{N})$, where $\sigma_{u}^{2}$ is the variance of $\left(u_{k}\right)_{k \in \mathbb{N}}$. For the proposed schemes and the UW-PSP, the projection in (6) is

\footnotetext{
${ }^{7}$ The speech sample is provided by "Special Research Project of the Typological Investigation into Languages \& Cultures of the East \& West (LACE)" in University of Tsukuba, Japan.
} 


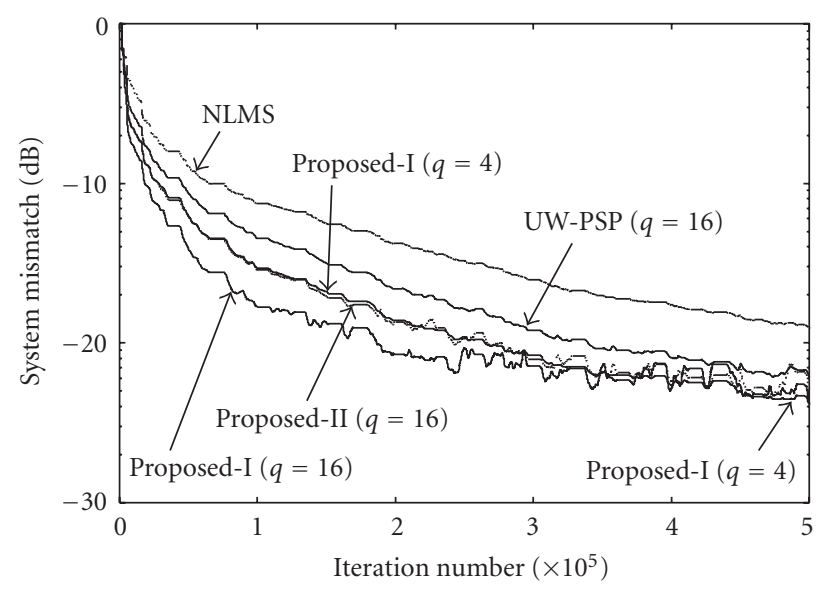

(a)

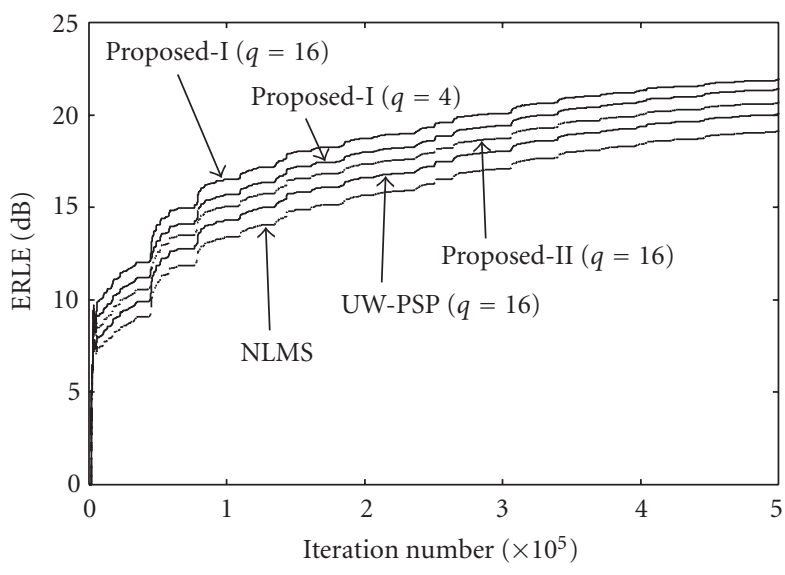

(b)

FIGURE 7: Proposed schemes versus UW-PSP for $r=1$ and $\lambda_{k}=0.4$ under SNR $=25 \mathrm{~dB}$. For a comparison, the performance of NLMS (a special case of the proposed method for $q=1$ ) is shown for $\lambda_{k}=0.2$.

regularized as

$$
\begin{aligned}
P_{H_{l}^{-}\left(\mathbf{h}_{k}\right)}^{(\delta)}\left(\mathbf{h}_{k}\right) & \\
: & = \begin{cases}\mathbf{h}_{k}-\frac{g_{l}\left(\mathbf{h}_{k}\right)}{\left\|\nabla g_{l}\left(\mathbf{h}_{k}\right)\right\|^{2}+\delta} \nabla g_{l}\left(\mathbf{h}_{k}\right) & \text { if } \mathbf{h}_{k} \notin H_{\iota}^{-}\left(\mathbf{h}_{k}\right), \\
\mathbf{h}_{k} & \text { otherwise, }\end{cases}
\end{aligned}
$$

where $\delta$ is set to $1.0 \times 10^{-6}$. In addition to the regularization for numerical stability against poor excitation, while the signal power is less than a common threshold, we stop the update for all algorithms throughout the simulations (this is the reason of the observable flat intervals in the figures).

To measure the achievement level for echo-path identification as well as echo cancellation, the following criteria are evaluated:

$$
\begin{aligned}
& \operatorname{system~mismatch~}(k):=10 \log _{10} \frac{\left\|\mathbf{h}^{*}-\mathbf{h}_{k}\right\|^{2}}{\left\|\mathbf{h}^{*}\right\|^{2}}, \quad \forall k \in \mathbb{N}, \\
& \operatorname{ERLE}(k):=10 \log _{10} \frac{\sum_{i=1}^{k} z_{i}^{2}}{\sum_{i=1}^{k}\left(z_{i}-\left\langle\mathbf{u}_{i}, \mathbf{h}_{i}\right\rangle\right)^{2}}, \quad \forall k \in \mathbb{N} .
\end{aligned}
$$

Simulations are conducted under several conditions.

\subsection{Proposed schemes versus UW-PSP with different $q$}

First, we examine the performance of the proposed schemes and the UW-PSP with $\left(\left|\ell_{k}^{(\mathrm{c})}\right|=\left|\boldsymbol{\ell}_{k}^{(\mathrm{p})}\right|=\right) q=4,16$ in Figure 7. For a comparison, the curve of NLMS with the step size $\lambda_{k}=$ 0.2 is drawn, which is a special case of POWER I for $q=$ $1, r=1, \rho=0, \lambda_{k}=0.4, \ell_{k}^{(0)}=l_{k}^{(\mathrm{c})}=\{k\}, \ell_{k}^{(1)}=\{(k, k)\}$ $(M=1)$, and $\ell_{k}^{(\mathrm{p})}=\varnothing$. For the proposed schemes, we set $\lambda_{k}=0.4(\forall k \in \mathbb{N}), r=1$, and $\rho=\max \left\{(r-2) \sigma^{2}, 0\right\}=0$, see Section 3.2 and [28]. The control sequences for POWER I are designed in the same manner as shown in Figure 4.
For POWER II and the UW-PSP, the curves of $q=4$ are omitted for visual clarity, since the difference between $q=4$ and $q=16$ is not significant. Referring to Figure 7, we see that the increase of $q$ for POWER I significantly improves the convergence speed without serious degradation in steady-state performance in both criteria. We also see that POWER I for $q=4$ exhibits faster convergence than the UWPSP for $q=16$. The above observation suggests that weight design is the key to attain better performance by increasing $q$.

\subsection{APA-based method with different $r$}

Next, we examine the performance of the APA for $r=$ $2,4,8,16$ in Figure 8, where $r$ is the dimension of affine projection (see Section 3.2). The APA-based method using data from one state of inputs at each iteration is referred to as "APA-I." The step size for $r=2$ is set to $\lambda_{k}=0.2$ for better performance. For $r=4,8,16$, two step sizes are employed; one is fixed to $\lambda_{k}=0.2$ (the same step size as $r=2$ ), for all $r$, and the other is individually tuned, for each $r$, so that the steady-state performance in system mismatch is almost the same as $r=2$ with $\lambda_{k}=0.2$.

Referring to Figure 8, the increase of $r$ for the APA-I raises the initial convergence speed at the expense of serious degradation in the steady-state performance in system mismatch, which causes gain loss in ERLE especially for $r=$ 8,16 . For the tuned step size, on the other hand, no distinct difference is observed among all $r$ in system mismatch, since, for large $r$, the small step size for good steady-state performance decreases the initial convergence speed. Comparing Figure 8 with Figure 7, it is seen that POWER I successfully alleviates the tradeoff problem between convergence speed and steady-state performance.

It should be remarked that these results do not contradict the results in other publications as mentioned below. Under high-SNR situations, it is reported that the increase of $r$ in the APA raises the speed of convergence, especially for highly 


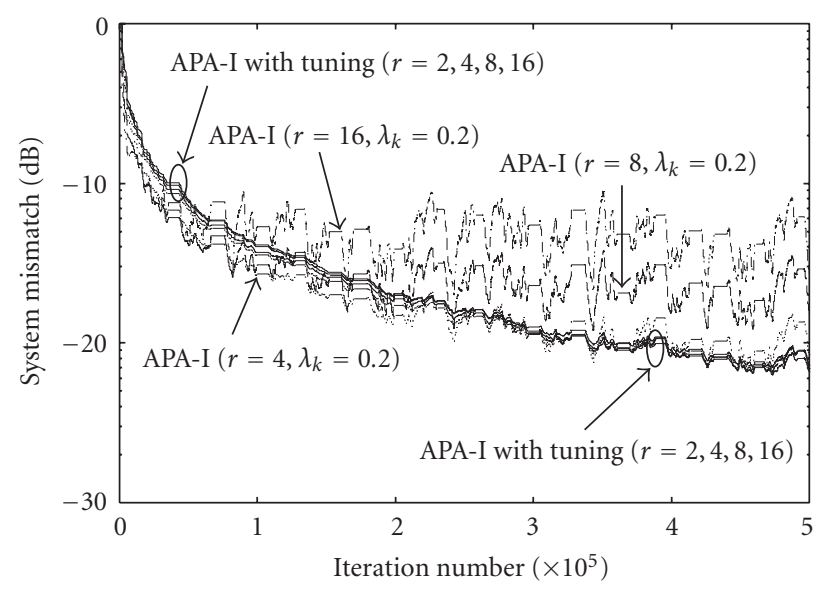

(a)

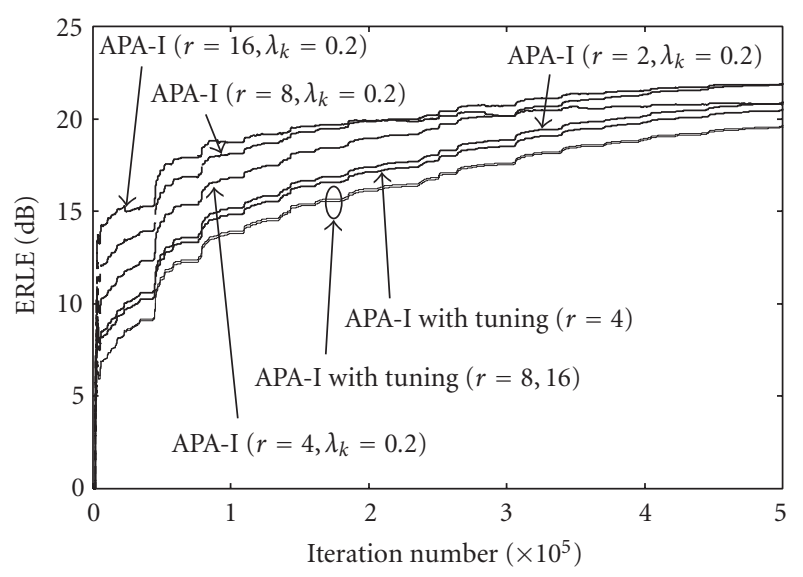

(b)

FIGURE 8: APA-I for $r=2,4,8,16$ under SNR $=25 \mathrm{~dB}$. For $r=2$, we set $\lambda_{k}=0.2$. For $r=4,8,16$, we use the same step size $\lambda_{k}=0.2$ and individually tuned one; $\lambda_{k}=0.1$ for $r=4, \lambda_{k}=0.04$ for $r=8$, and $\lambda_{k}=0.022$ for $r=16$.

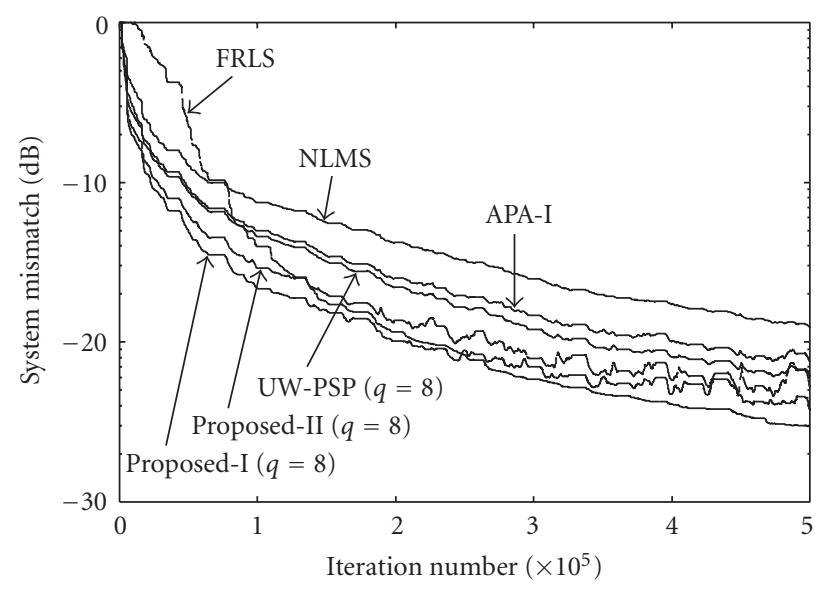

(a)

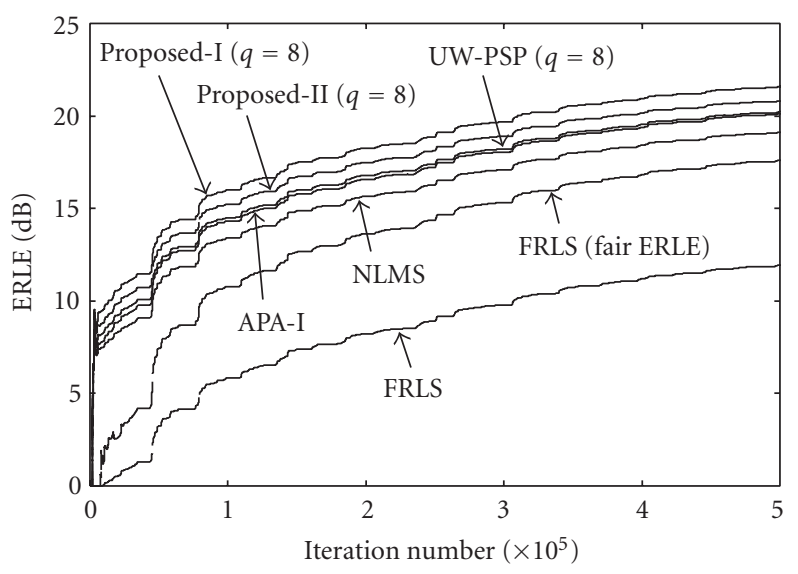

(b)

FIGURE 9: Proposed schemes versus UW-PSP, NLMS, and APA-I under SNR $=25 \mathrm{~dB}$. For the NLMS, $\lambda_{k}=0.2$. For the APA-I, $r=2$ and $\lambda_{k}=0.15$. For the FRLS, $\gamma=1-1 / 18 N$. For the proposed schemes and the UW-PSP, $r=1, \lambda_{k}=0.4$, and $q=8$.

colored excited input signals, without severely deteriorating the steady-state performance (see, e.g., [48-51]). Under lowSNR situations, on the other hand, it is theoretically verified that the increase of $r$ in the APA decreases the membership probability $\mathbf{h}^{*} \in V_{k}$ (especially for $r \geq 3$, Prob $\left(\mathbf{h}^{*} \in V_{k}\right) \approx$ 0 ) [28, Section III], which causes serious noise sensitivity of the APA for $r \geq 3$ (see also Section 3.2).

\subsection{Proposed schemes versus UW-PSP, APA, NLMS, and FRLS with fixed and time-varying echo paths}

The proposed schemes are now compared with the UW-PSP, APA-I, NLMS, and FRLS algorithms in Figures 9 and 10. For the proposed schemes and the UW-PSP, the parameters are exactly the same as in Figure 7 except that $q=8$. For the
NLMS, the step size is set to 0.2 to attain better steady-state performance. For the APA-I, we set $r=2$ and $\lambda_{k}=0.15$ so that the initial convergence speed is the same as the UW-PSP. For the FRLS, the forgetting factor is set to $\gamma=1-1 / 18 N$ for the best performance among our experiments. We remark that the FRLS algorithm exhibits severe sensitivity against the choice of the forgetting factor or the regularization parameter $\xi_{k}^{2}$; for example, once we tried to employ $\gamma=1-1 / 15 N$, the speed of convergence was a little faster but the filter diverged around the iteration number 500000. In this simulation, although the steady-state performance is not the same as the proposed schemes, the parameters are tuned carefully.

Figure 9 depicts the results under the condition of fixed echo paths. We observe that the proposed schemes attain 


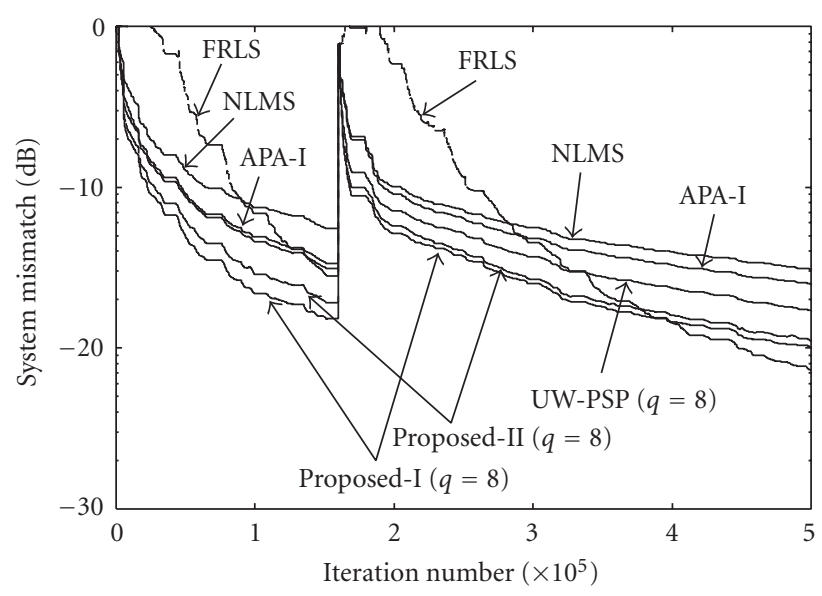

(a)

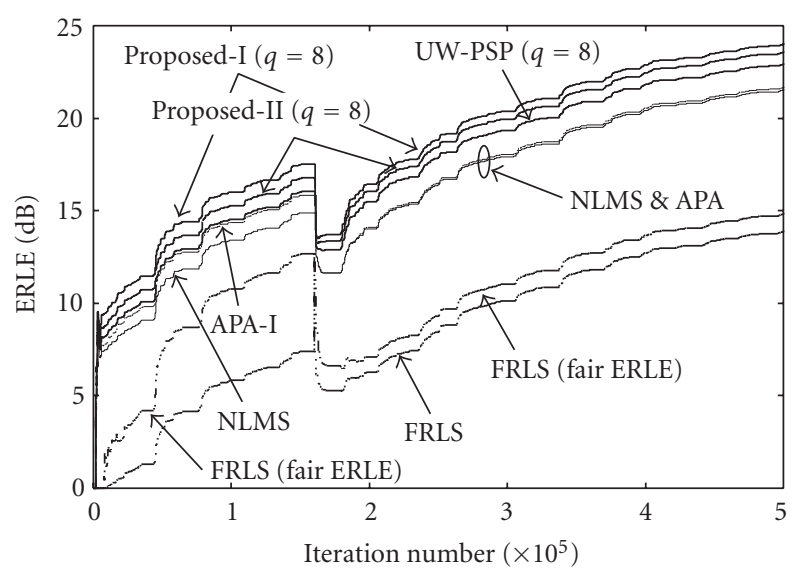

(b)

FIgUre 10: Proposed schemes versus UW-PSP, NLMS, and APA-I with the echo paths changed at the iteration number $1.6 \times 10^{5}$. The other conditions are the same as in Figure 9.

TABLe 1: Time needed to achieve the system mismatch level of $-20 \mathrm{~dB}$.

\begin{tabular}{l|cccccc}
\hline Method & POWER I & POWER II & UW-PSP & FRLS & APA-I & NLMS \\
\hline Second & 25 & 31 & 43 & 28 & 50 & 75 \\
\hline
\end{tabular}

much faster convergence as well as better steady-state performance than the NLMS, APA-I, and FRLS algorithms. The time for POWER I to achieve the system mismatch level of $-20 \mathrm{~dB}$ is approximately 25 second. The time for each algorithms is summarized in Table 1. POWER I is approximately 45 second, 25 second, and 3 second faster than the NLMS, the APA-I, and the FRLS, respectively. Figure 10 depicts the results under the condition where the echo-paths are changed at the iteration number $1.6 \times 10^{5}$. We see that the proposed schemes exhibit excellent tracking behavior against echo path variation. In Figures 9 and 10, the FRLS exhibits poor ERLE performance due to the observable instability in system mismatch at the beginning of adaptation. For fairness, we also draw the curves of the FRLS in a different ERLE criterion in which the summations are taken (not from $i=1$ but) from the moment when its system mismatch becomes less than $0 \mathrm{~dB}$ (this new ERLE criterion is referred to as "fair ERLE").

It is reported that the RLS algorithm exhibits, besides its high computational complexity, an instability issue especially for (nonstationary) speech signals, and thus has been discouraged to be used in acoustic echo cancellation [11, page 77]. Also the FRLS algorithms inherit the instability issue, as pointed out in a considerable amount of literature, for example, [7, page 40], [52-55]. Moreover, the observable slow initial convergence of the FRLS stems from the same reason as its tracking inferiority, under nonstationary environments, to the LMS-type algorithms, as remarked, for example, in $[44,56,57]$.

\subsection{Proposed schemes versus APA with simultaneous use of data from two states}

Finally, POWER I is compared, in Figure 11, with the remaining possibility to resolve the zigzag loss (see Section 1), that is, the APA with simultaneous use of data from two states of inputs. Namely, for all $k \geq Q / 2+r / 2, \widetilde{\mathbf{e}}_{k}(\mathbf{h}):=\widetilde{\mathbf{U}}_{k}^{T} \mathbf{h}-\widetilde{\mathbf{d}}_{k}$ is used to define $V_{k}$ (see Section 3.2) instead of $\mathbf{e}_{k}(\mathbf{h})$, where $\widetilde{\mathbf{U}}_{k}:=\left[\mathbf{u}_{k} \cdots \mathbf{u}_{k-r / 2+1} \mathbf{u}_{k-Q / 2} \cdots \mathbf{u}_{k-Q / 2-r / 2+1}\right] \in \mathbb{R}^{2 N \times r}$ and $\tilde{\mathbf{d}}_{k}:=\widetilde{\mathbf{U}}_{k}^{T} \mathbf{h}^{*}+\tilde{\mathbf{n}}_{k} \in \mathbb{R}^{r}$ with $\tilde{\mathbf{n}}_{k}:=\left[n_{k}, \ldots, n_{k-r / 2+1}, n_{k-Q / 2}\right.$, $\left.\ldots, n_{k-Q / 2-r / 2+1}\right]^{T}$. This new APA method is referred to as "APA-II." For the proposed scheme, the parameters are the same as in Figure 7 (or in Figure 9) for $q=4,8$. For the APAII, for fairness, $r=8,16$ are employed with the tuned step sizes $\lambda_{k}=0.04,0.022$, respectively. For a comparison, the curves of APA-I and II with $r=2$ and $\lambda_{k}=0.2$ are also drawn.

In Figure 11, we observe that the proposed scheme achieves faster initial convergence and better steady-state performance than the APA-II in both criteria. Moreover, for the APA-II, the increase of $r$ improves the initial convergence speed at the expense of unignorable deterioration in ERLE. On the other hand, for the proposed scheme, the increase of $q$ improves the performance in both criteria, as also shown in Figure 7.

\section{CONCLUSION}

This paper has presented a class of efficient fast stereophonic acoustic echo cancelling schemes based on the POWER weighting technique. The proposed schemes successfully accelerate the convergence with keeping linear complexity and good steady-state performance. Numerical examples have verified the efficacy of the proposed schemes. The results of the extensive simulations suggest that the POWER technique is significantly effective especially for the challenging stereophonic echo cancelling problem. 


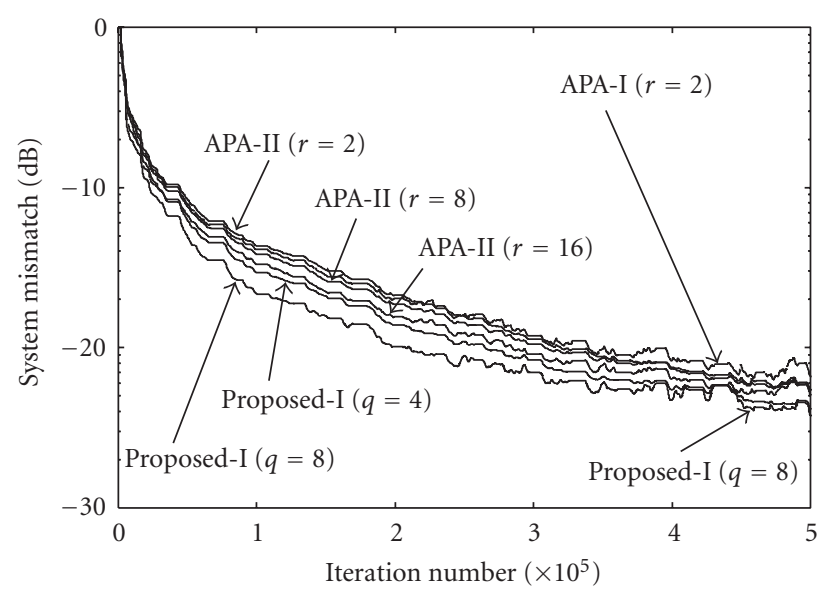

(a)

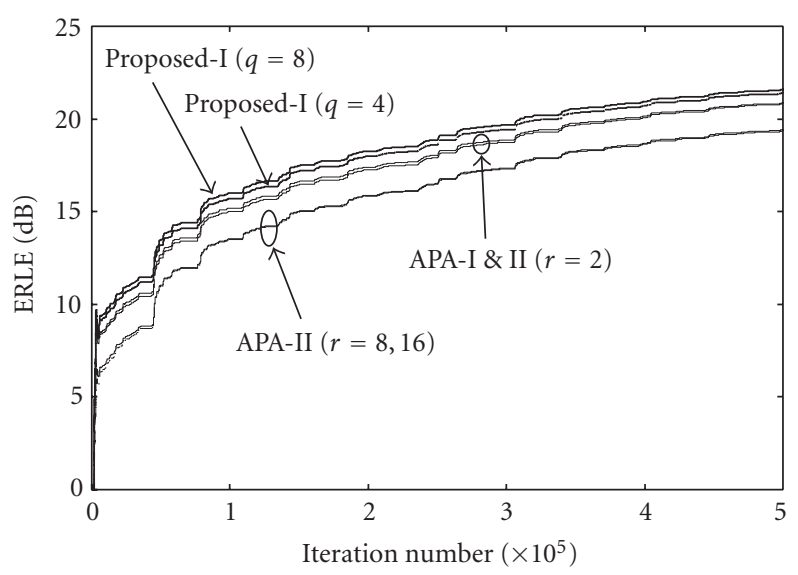

(b)

FIgUre 11: Proposed schemes $(q=4,8)$ versus APA-II $(r=2,8,16)$ under SNR $=25 \mathrm{~dB}$. For the proposed schemes, we employ the same parameters as in Figure 7. For APA-II, $\lambda_{k}=0.2,0.04,0.022$ for $r=2,8,16$, respectively. For APA-I, $r=2$ and $\lambda_{k}=0.2$.

\section{APPENDICES}

\section{A. PREPROCESSING TECHNIQUES}

As stated in Section 2.2, the difficulty of nonuniqueness has been known to be inherent in the SAEC problem. To alleviate this difficulty, several excellent preprocessing techniques ${ }^{8}$ were proposed; half-wave rectifier [5] (see [22] for an improved version), comb filtering $[3,17]$, additive noise $[18,19]$, and time-varying filtering [14-16], (see [21] for a generalized version of [14]); another nonlinear preprocessing technique is also proposed in [20]. Indeed, efficacy of several nonlinear preprocessing techniques was quantified with mutual coherence of the stereo inputs [62].

Figure 12 illustrates a simple example of the preprocessing unit generating two states of inputs (see also Figure 1). In $[14,15]$, it is reported that periodic one-sample delays, in one side of stereo inputs (i.e., $\mathbf{u}_{k}^{(1)}$ in Figure 1), realize accurate echo-path identification without audible degradation in speech. Since $\mathbf{u}_{k}^{(1)}$ is generated by convolution of the talker's speech $s_{k}$ with the transmission path $\boldsymbol{\theta}_{(1)}$, the periodic delays virtually give one-sample shift to $\boldsymbol{\theta}_{(1)}$. In other words, the preprocessing technique introduces a slightly modified state of input and alternates two $9{ }^{9}$ (modified and nonmodified) states of inputs periodically, leading to alternation of two states of transmission path, say $\boldsymbol{\theta}_{(1)}$ and $\tilde{\boldsymbol{\theta}}_{(1)}$. As a result, since the solution set depends on transmission paths as mentioned above, two slightly different solution sets, $\mathcal{V}\left(\boldsymbol{\theta}_{(1)}\right)$ and $\mathcal{V}\left(\tilde{\boldsymbol{\theta}}_{(1)}\right)$ (corresponding to $\mathcal{V}$ and $\tilde{\mathcal{V}}$ in Figure 2, resp.), are generated alternately.

\footnotetext{
${ }^{8}$ Some nonpreprocessing techniques were also proposed with an advantage of no degradation in input signals [58-61], however, their tracking speed of echo paths is somewhat inferior to some preprocessing techniques.

${ }^{9}$ Although the number of states could be generalized to more than two by generating more than one modified state, we adopt two states for simplicity.
}

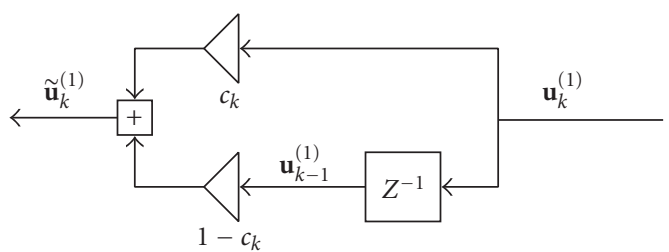

FIgURE 12: A preprocessing unit called input sliding. The factor $c_{k}$ slides between 0 and 1 periodically, and thus, $\widetilde{\mathbf{u}}_{k}^{(1)}:=c_{k} \mathbf{u}_{k}^{(1)}+(1-$ $\left.c_{k}\right) \mathbf{u}_{k-1}^{(1)}$ is a periodically delayed version of $\mathbf{u}_{k}^{(1)}$.

\section{B. SAEC SCHEME PROPOSED IN [23]}

Scheme 3 (see [23]). Suppose that a sequence of closed convex sets $\left(C_{l}(\rho)\right)_{l \in \ell} \subset \mathscr{H}$ is defined as in (4), where $\ell:=$ $\bigcup_{k \in \mathbb{N}}\left(l_{k}^{(\mathrm{c})} \cup \ell_{k}^{(\mathrm{p})}\right)$. Let $\mathbf{h}_{0} \in \mathscr{H}$ be an arbitrarily chosen initial vector. Then, define a sequence of filtering vectors $\left(\mathbf{h}_{k}\right)_{k \in \mathbb{N}} \subset \mathscr{H}$ by

$$
\mathbf{h}_{k+1}:=\mathbf{h}_{k}+\lambda_{k} \mathcal{M}_{k}\left(\sum_{\iota \in \ell_{k}^{(c)} \cup \ell_{k}^{(\mathrm{p})}} w_{\iota}^{(k)} P_{H_{\iota}^{-}\left(\mathbf{h}_{k}\right)}\left(\mathbf{h}_{k}\right)-\mathbf{h}_{k}\right),
$$

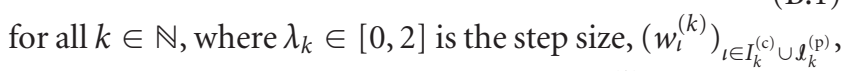
for all $k \in \mathbb{N}$, are the weights satisfying $w_{l}^{(k)} \in[0,1]$, and $\sum_{\iota \in \ell_{k}^{(\mathrm{c})} \cup \ell_{k}^{(\mathrm{p})}} w_{l}^{(k)}=1$, and

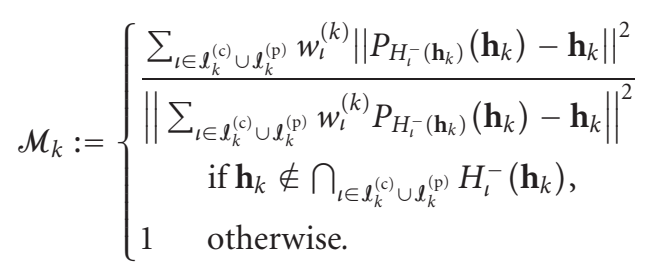

If $w_{l}^{(k)}=1 / 2 q\left(\forall \iota \in \ell_{k}^{(\mathrm{c})} \cup \ell_{k}^{(\mathrm{p})}, \forall k \in \mathbb{N}\right)$, the scheme is called UW-PSP. 


\section{COMPUTATION OF $\mathcal{P}(\mathbf{s}, \mathbf{a}, \mathbf{b})$ AND PAIRWISE-OPTIMALITY}

The following proposition gives an efficient way to calculate $\mathcal{P}(\mathbf{s}, \mathbf{a}, \mathbf{b})$ with given $(\mathbf{s}, \mathbf{a}, \mathbf{b}) \in \mathscr{H}^{3}$.

Proposition 1 (projection onto intersection of two halfspaces [32]). Given $(\mathbf{s}, \mathbf{a}, \mathbf{b}) \in \mathscr{H}^{3}$ s.t. $\Pi^{-}(\mathbf{s}, \mathbf{a}) \cap \Pi^{-}(\mathbf{s}, \mathbf{b}) \neq$ $\varnothing$, let $\xi:=\|\mathbf{a}-\mathbf{s}\|^{2}, \zeta:=\|\mathbf{b}-\mathbf{s}\|^{2}$, and $\eta:=\langle\mathbf{a}-\mathbf{s}, \mathbf{b}-\mathbf{s}\rangle$. Then,

$$
\mathcal{P}(\mathbf{s}, \mathbf{a}, \mathbf{b})=\mathbf{s}+\mu^{*}\left\{\omega^{*} \mathbf{a}+\left(1-\omega^{*}\right) \mathbf{b}-\mathbf{s}\right\},
$$

where

$$
\begin{aligned}
\mu^{*} & := \begin{cases}1 & \text { if } \eta \geq \xi \text { or } \eta \geq \zeta, \\
\frac{2 \xi \zeta-(\xi+\zeta) \eta}{\xi \zeta-\eta^{2}} & \text { if } \eta<\min \{\xi, \zeta\},\end{cases} \\
\omega^{*}: & = \begin{cases}1 & \text { if } \eta \geq \zeta, \\
0 & \text { if } \zeta>\eta \geq \xi, \\
\frac{\zeta(\xi-\eta)}{2 \xi \zeta-(\xi+\zeta) \eta} & \text { if } \eta<\min \{\xi, \zeta\} .\end{cases}
\end{aligned}
$$
by

Let us define the operator $\mathcal{Q}:[0,1] \times[0, \infty) \times \mathscr{H}^{3} \rightarrow \mathscr{H}$

$$
\mathcal{Q}(\omega, \mu, \mathbf{s}, \mathbf{a}, \mathbf{b}):=\mathbf{s}+\mu\{\omega \mathbf{a}+(1-\omega) \mathbf{b}-\mathbf{s}\} .
$$

By (C.1) and (C.3), we see that $\mathcal{P}(\mathbf{s}, \mathbf{a}, \mathbf{b})=\mathcal{Q}\left(\omega^{*}, \mu^{*}, \mathbf{s}\right.$, a, b). An optimality of $\omega^{*}$ and $\mu^{*}$ is shown below.

Proposition 2 (optimality of $\omega^{*}$ and $\mu^{*}$ [32]). Given (s, $\mathbf{a}, \mathbf{b}) \in \mathscr{H}^{3}$ s.t. $\Pi^{-}(\mathbf{s}, \mathbf{a}) \cap \Pi^{-}(\mathbf{s}, \mathbf{b}) \neq \varnothing$, let $\phi(\omega, \mu, \mathbf{z}):=$ $\|\mathbf{s}-\mathbf{z}\|^{2}-\|\mathcal{Q}(\omega, \mu, \mathbf{s}, \mathbf{a}, \mathbf{b})-\mathbf{z}\|^{2}$. Then, $\left(\omega^{*}, \mu^{*}\right)$ in Proposition 1 is optimal in the sense of

$$
\left(\omega^{*}, \mu^{*}\right) \in \underset{(\omega, \mu) \in[0,1] \times[0, \infty)}{\arg \max } \min _{\mathbf{z} \in \Pi^{-}(\mathbf{s , a}) \cap \Pi^{-}(\mathbf{s}, \mathbf{b})} \phi(\omega, \mu, \mathbf{z}) .
$$

Intuitively, $\left(\omega^{*}, \mu^{*}\right)$ achieves a worst-case optimization, or, in other words, (C.4) implies that $\left(\omega^{*}, \mu^{*}\right)$ is a solution to the max-min problem of maximizing, over $\omega$ and $\mu$, the minimum of $\phi(\omega, \mu, \mathbf{z})$ over $\mathbf{z}$. A geometric interpretation of Propositions 1 and 2 is given in Figure 13.

Another proposition is presented below to show an optimality of the weights realized by the proposed schemes.

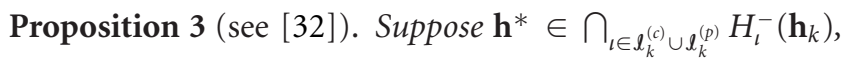
$k \in \mathbb{N}$. Then, the following hold:

(I) $\mathbf{h}^{*} \in \Pi^{-}\left(\mathbf{h}_{k}, \mathbf{h}_{k, l}^{(m)}\right), \forall \iota \in \boldsymbol{l}_{k}^{(m)}, \forall m \in\{1, \ldots, M-1\}$,

(II) $\mathbf{h}^{*} \in \Pi^{-}\left(\mathbf{h}_{k}, \mathbf{h}_{k}^{(c)}\right) \cap \Pi^{-}\left(\mathbf{h}_{k}, \mathbf{h}_{k}^{(p)}\right)$.

For POWER I, Propositions 2 and 3(I) imply that the direction of update is getting improved step by step through multiple stages, since the weights realized in each stage are

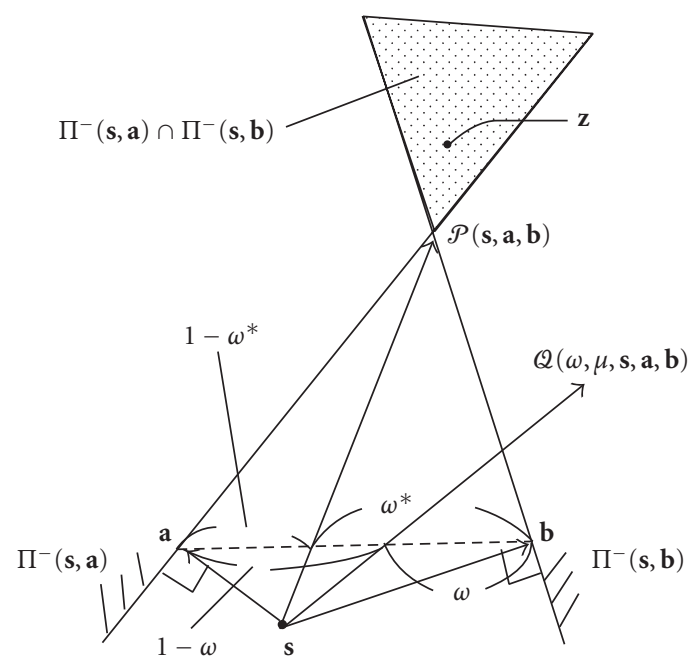

Figure 13: A geometric interpretation of Propositions 1 and 2. Let $\mathbf{h}_{k}:=\mathbf{s}$ and $\mathbf{h}_{k+1}:=\mathcal{Q}(\omega, \mu, \mathbf{s}, \mathbf{a}, \mathbf{b})$. Then, $\|\mathbf{s}-\mathbf{z}\|=\left\|\mathbf{h}_{k}-\mathbf{z}\right\|$ and $\|Q(\omega, \mu, \mathbf{s}, \mathbf{a}, \mathbf{b})-\mathbf{z}\|=\left\|\mathbf{h}_{k+1}-\mathbf{z}\right\|$ denote the distance to $\mathbf{z}\left[\in \Pi^{-}(\mathbf{s}, \mathbf{a}) \cap \Pi^{-}(\mathbf{s}, \mathbf{b})\right]$ from the filtering vector before and after update, respectively. Proposition 2 means that $\left(\omega^{*}, \mu^{*}\right)$ given in Proposition 1 maximizes $\min _{\mathbf{z} \in \Pi^{-}(\mathbf{s}, \mathbf{a}) \cap \Pi^{-}(\mathbf{s}, \mathbf{b})}\left\|\mathbf{h}_{k}-\mathbf{z}\right\|^{2}-\left\|\mathbf{h}_{k+1}-\mathbf{z}\right\|^{2}$.

optimal in the sense of a solution to a worst-case optimization problem. For POWER II, similarly, Propositions 2 and 3 (II) imply that the weights realized in the second stage are optimal.

\section{WEIGHT REALIZATION}

In this appendix, we show that POWER I and POWER II can be written in the form of the scheme proposed in [23], which is given in Appendix B. The weights realized by POWER I are given as follows.

Proposition 4 (weight realization by POWER I [32]). Let $\left(\mathbf{h}_{k}\right)_{k \in \mathbb{N}} \subset \mathcal{H}$ be a sequence of filtering vectors generated by Scheme 1. Then, $\mathbf{h}_{k+1}$ is represented in the form of Scheme 3 with $\left(w_{j}^{(k)}=w_{j, l, M}^{(k)}\right)_{j \in \ell_{k}^{(c)} \cup \ell_{k}^{(p)}}$, for all $k \in \mathbb{N}$, satisfying that $w_{j}^{(k)}>0$ and $\sum_{j \in \ell_{k}^{(c)} \cup l_{k}^{(p)}} w_{j}^{(k)}=1$, where $w_{j, l, M}^{(k)}$ is defined by the following simple recursive form: if $\eta_{k, l}^{(m)}=-\sqrt{\xi_{k, l}^{(m)} \zeta_{k, l}^{(m)}} \neq 0$, then $w_{j, l, m}^{(k)}=0$ $\left(\forall m=1,2, \ldots, M, \forall i \in l_{k}^{(m)}, \forall j \in l_{k}^{(c)} \cup l_{k}^{(p)}\right)$, otherwise,

$$
w_{j, \iota, 1}^{(k)}:= \begin{cases}\omega^{*} & \text { if } \iota=(j, \cdot), \\ 1-\omega^{*} & \text { if } \iota=(\cdot, j), \\ 0 & \text { otherwise, }\end{cases}
$$

$$
\begin{array}{r}
\forall j \in \ell_{k}^{(c)} \cup \ell_{k}^{(p)}, \quad \forall \iota \in l_{k}^{(1)}, \\
w_{j, \iota, m}^{(k)}:=\frac{\omega^{*} \mu_{L}^{*} w_{j, l_{L}, m-1}^{(k)}+\left(1-\omega^{*}\right) \mu_{R}^{*} w_{j, l_{R}, m-1}^{(k)}}{\omega^{*} \mu_{L}^{*}+\left(1-\omega^{*}\right) \mu_{R}^{*}}, \\
\forall j \in l_{k}^{(c)} \cup l_{k}^{(p)}, \quad \forall \iota=\left(\iota_{L}, \iota_{R}\right) \in l_{k}^{(m)}, \quad \forall m=2,3, \ldots, M,
\end{array}
$$


where $\omega^{*}$ for $w_{j, l, m}^{(k)}(\forall m=1,2, \ldots, M)$ denotes the weight to calculate $\mathbf{h}_{k, l}^{(m)}\left(=\mathcal{P}\left(\mathbf{h}_{k}, \mathbf{h}_{k, l_{1}}^{(m-1)}, \mathbf{h}_{k, l_{2}}^{(m-1)}\right)\right)$ and $\mu_{i}^{*}(i=L, R)$ for $w_{j, l, m}^{(k)}(\forall m=2,3, \ldots, M)$ denotes the relaxation parameter to calculate $\mathbf{h}_{k, l_{i}}^{(m-1)}$ (see Proposition 1).

Remark 2. It may happen that $w_{j, l, M}^{(k)}=0$, for all $j \in \ell_{k}^{(\mathrm{c})} \cup$ $\iota_{k}^{(\mathrm{p})}$, when, for instance, $\eta_{k, l}^{(1)}=-\sqrt{\xi_{k, l}^{(1)} \zeta_{k, l}^{(1)}} \neq 0$, for all $\iota=$ $\left(\iota_{1}, \iota_{2}\right) \in \ell_{k}^{(1)}$. However, noting that $\eta_{k, l}^{(1)}=-\sqrt{\xi_{k, l}^{(1)} \zeta_{k, l}^{(1)}} \neq 0 \Leftrightarrow$ $\mathbf{h}_{k, l_{1}}^{(0)}-\mathbf{h}_{k}=\delta\left(\mathbf{h}_{k, l_{2}}^{(0)}-\mathbf{h}_{k}\right) \neq \mathbf{0}$, there exists $\delta<0$ (see [32]), such a case can be neglected. This is because $\mathbf{h}_{k, l_{1}}^{(0)}$ and $\mathbf{h}_{k, l_{2}}^{(0)}$ provide inconsistent information, which implies that the data are not reliable. All the cases when it happens that $w_{j, l, M}^{(k)}=0$, for all $j \in \ell_{k}^{(\mathrm{c})} \cup \ell_{k}^{(\mathrm{p})}$, are caused by frequent occurrence of $\eta_{k, \iota}^{(1)}=-\sqrt{\xi_{k, \iota}^{(1)} \zeta_{k, \iota}^{(1)}} \neq 0$, hence such cases are of no importance. Except for this kind of situations, the overall weights realized by POWER I satisfies the conditions imposed on $w_{\iota}^{(k)}$ in Scheme 3. lows.

Next, the weight realization by POWER II is given as fol-

Proposition 5 (weight realization by POWER II [32]). Let $\left(\mathbf{h}_{k}\right)_{k \in \mathbb{N}} \subset \mathcal{H}$ be a sequence of filtering vectors generated by Scheme 2. Then, $\mathbf{h}_{k+1}$ is represented in the form of Scheme 3 with the weights as follows: if $\eta_{k}=-\sqrt{\xi_{k} \zeta_{k}} \neq 0$, then $w_{l}^{(k)}=0$, for all $\iota \in l_{k}^{(c)} \cup \ell_{k}^{(p)}$, otherwise,

$$
w_{\iota}^{(k)}:= \begin{cases}\frac{\omega_{k}^{*} \mathcal{M}_{k}^{(c)} w_{k}^{(c)}}{\alpha_{k}} & \forall \iota \in \mathcal{l}_{k}^{(c)}, \\ \frac{\left(1-\omega_{k}^{*}\right) \mathcal{M}_{k}^{(p)} w_{k}^{(p)}}{\alpha_{k}} & \forall \iota \in l_{k}^{(p)},\end{cases}
$$

where $\alpha_{k}:=\left|\ell_{k}^{(c)}\right| \omega_{k}^{*} \mathcal{M}_{k}^{(c)} w_{k}^{(c)}+\left|\boldsymbol{\ell}_{k}^{(p)}\right|\left(1-\omega_{k}^{*}\right) \mathcal{M}_{k}^{(p)} w_{k}^{(p)}$ and $\omega_{k}^{*}$ is the weight to calculate $\mathcal{P}\left(\mathbf{h}_{k}, \mathbf{h}_{k}^{(c)}, \mathbf{h}_{k}^{(p)}\right)$ (see Proposition 1).

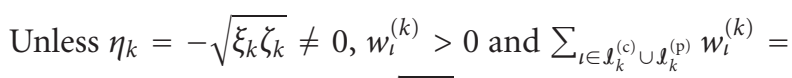

1. Note that the case of $\eta_{k}=-\sqrt{\xi_{k} \zeta_{k}} \neq 0$ is of no importance for the same reason as stated in Remark 2.

\section{ACKNOWLEDGMENTS}

The authors would like to express their deep gratitude to Professor K. Sakaniwa of Tokyo Institute of Technology and Professor A. Hirano of Kanazawa University for fruitful discussions. They are also grateful to Professor S. Furui of Tokyo Institute of Technology for his kind advice on speech samples, which surely promoted our experiments. Finally, they would like to thank the anonymous reviewers for providing greatly constructive comments.

\section{REFERENCES}

[1] T. Fujii and S. Shimada, "A note on multi-channel echo cancellers," CS84-178, IEICE, January 1985, in Japanese.

[2] M. M. Sondhi and D. R. Morgan, "Acoustic echo cancellation for stereophonic teleconferencing," in Proceedings of the IEEE Workshop on Applications of Signal Processing to Audio and Acoustics (ASSP '91), pp. 141-142, October 1991.

[3] M. M. Sondhi, D. R. Morgan, and J. L. Hall, "Stereophonic acoustic echo cancellation-an overview of the fundamental problem," IEEE Signal Processing Letters, vol. 2, no. 8, pp. 148151, 1995.

[4] J. Benesty, F. Amand, A. Gilloire, and Y. Grenier, "Adaptive filtering algorithms for stereophonic acoustic echo cancellation," in Proceedings of the International Conference on Acoustics, Speech, and Signal Processing (ICASSP '95), vol. 5, pp. 3099-3102, Detroit, Mich, USA, May 1995.

[5] J. Benesty, D. R. Morgan, and M. M. Sondhi, "A better understanding and an improved solution to the specific problems of stereophonic acoustic echo cancellation," IEEE Transactions on Speech and Audio Processing, vol. 6, no. 2, pp. 156-165, 1998.

[6] T. Gänsler and J. Benesty, "Stereophonic acoustic echo cancellation and two-channel adaptive filtering: an overview," International Journal of Adaptive Control and Signal Processing, vol. 14, no. 6, pp. 565-586, 2000.

[7] S. L. Gay and J. Benesty, Eds., Acoustic Signal Processing for Telecommunication, Kluwer Academic, Boston, Mass, USA, 2000.

[8] P. Eneroth, S. L. Gay, T. Gänsler, and J. Benesty, "A real-time implementation of a stereophonic acoustic echo canceler," IEEE Transactions on Speech and Audio Processing, vol. 9, no. 5, pp. 513-523, 2001.

[9] T. Gänsler and J. Benesty, "Multichannel acoustic echo cancellation: what's new?" in Proceedings of the 7th International Workshop on Acoustic Echo and Noise Control (IWAENC '01), Darmstadt, Germany, September 2001.

[10] K. Ikeda and R. Sakamoto, "Convergence analyses of stereo acoustic echo cancelers with preprocessing," IEEE Transactions on Signal Processing, vol. 51, no. 5, pp. 1324-1334, 2003.

[11] J. Benesty and Y. Huang, Eds., Adaptive Signal Processing: Applications to Real-World Problems, Springer, Berlin, Germany, 2003.

[12] A. Sugiyama, A. Hirano, and K. Nakayama, "Acoustic echo cancellation for conference systems," in Proceedings of the European Signal Processing Conference (EUSIPCO '04), pp. 17-20, Vienna, Austria, September 2004.

[13] H. Buchner, J. Benesty, and W. Kellermann, "Generalized multichannel frequency-domain adaptive filtering: efficient realization and application to hands-free speech communication," Signal Processing, vol. 85, no. 3, pp. 549-570, 2005.

[14] Y. Joncour and A. Sugiyama, "A stereo echo canceler with preprocessing for correct echo-path identification," in Proceedings of the IEEE International Conference on Acoustics, Speech, and Signal Processing (ICASSP '98), vol. 6, pp. 3677-3680, Seattle, Wash, USA, May 1998.

[15] A. Sugiyama, Y. Joncour, and A. Hirano, "A stereo echo canceler with correct echo-path identification based on an inputsliding technique," IEEE Transactions on Signal Processing, vol. 49, no. 11, pp. 2577-2587, 2001.

[16] M. Ali, "Stereophonic acoustic echo cancellation system using time-varying all-pass filtering for signal decorrelation," in Proceedings of the IEEE International Conference on Acoustics, Speech, and Signal Processing (ICASSP '98), vol. 6, pp. 36893692, Seattle, Wash, USA, May 1998. 
[17] J. Benesty, D. R. Morgan, J. L. Hall, and M. M. Sondhi, "Stereophonic acoustic echo cancellation using nonlinear transformations and comb filtering," in Proceedings of the IEEE International Conference on Acoustics, Speech, and Signal Processing (ICASSP '98), vol. 6, pp. 3673-3676, Seattle, Wash, USA, May 1998.

[18] T. Gänsler and P. Eneroth, "Influence of audio coding on stereophonic acoustic echo cancellation," in Proceedings of the IEEE International Conference on Acoustics, Speech, and Signal Processing (ICASSP '98), vol. 6, pp. 3649-3652, Seattle, Wash, USA, May 1998.

[19] A. Gilloire and V. Turbin, "Using auditory properties to improve the behaviour of stereophonic acoustic echo cancellers," in Proceedings of the IEEE International Conference on Acoustics, Speech, and Signal Processing (ICASSP '98), vol. 6, pp. 3681-3684, Seattle, Wash, USA, May 1998.

[20] S. Shimauchi, Y. Haneda, S. Makino, and Y. Kaneda, "New configuration for a stereo echo canceller with nonlinear preprocessing," in Proceedings of the IEEE International Conference on Acoustics, Speech, and Signal Processing (ICASSP' 98), vol. 6, pp. 3685-3688, Seattle, Wash, USA, May 1998.

[21] A. Hirano, K. Nakayama, and K. Watanabe, "Convergence analysis of stereophonic echo canceller with pre-processingrelation between pre-processing and convergence," in Proceedings of the IEEE International Conference on Acoustics, Speech, and Signal Processing (ICASSP '99), vol. 2, pp. 861-864, Phoenix, Ariz, USA, March 1999.

[22] T. Gänsler and J. Benesty, "New insights into the stereophonic acoustic echo cancellation problem and an adaptive nonlinearity solution," IEEE Transactions on Speech and Audio Processing, vol. 10, no. 5, pp. 257-267, 2002.

[23] M. Yukawa and I. Yamada, "Efficient adaptive stereo echo canceling schemes based on simultaneous use of multiple state data," IEICE Transactions on Fundamentals of Electronics, Communications and Computer Sciences, vol. E87-A, no. 8, pp. 1949-1957, 2004.

[24] I. Yamada, "Adaptive projected subgradient method: unified view for projection based adaptive algorithms," Journal of IEICE, vol. 86, no. 8, pp. 654-658, 2003.

[25] I. Yamada and N. Ogura, "Adaptive projected subgradient method for asymptotic minimization of sequence of nonnegative convex functions," Numerical Functional Analysis and Optimization, vol. 25, no. 7\&8, pp. 593-617, 2004.

[26] R. L. G. Cavalcante, I. Yamada, and K. Sakaniwa, "A fast blind MAI reduction based on adaptive projected subgradient method," IEICE Transactions on Fundamentals of Electronics, Communications and Computer Sciences, vol. E87-A, no. 8, pp. 1973-1980, 2004.

[27] M. Yukawa, R. L. G. Cavalcante, and I. Yamada, "Efficient blind MAI suppression in DS/CDMA systems by embedded constraint parallel projection techniques," IEICE Transactions on Fundamentals of Electronics, Communications and Computer Sciences, vol. E88-A, no. 8, pp. 2062-2071, 2005.

[28] I. Yamada, K. Slavakis, and K. Yamada, "An efficient robust adaptive filtering algorithm based on parallel subgradient projection techniques," IEEE Transactions on Signal Processing, vol. 50, no. 5, pp. 1091-1101, 2002.

[29] F. R. Deutsch, Best Approximation in Inner Product Spaces, Springer, Berlin, Germany, 2001.

[30] Y. Censor and S. A. Zenios, Parallel Optimization: Theory, Algorithms, and Applications, Oxford University Press, New York, NY, USA, 1997.
[31] M. Yukawa and I. Yamada, "Acceleration of adaptive parallel projection algorithms by pairwise optimal weight realization," in Proceedings of the 12th European Signal Processing Conference (EUSIPCO '04), pp. 713-716, Vienna, Austria, September 2004.

[32] M. Yukawa and I. Yamada, "Pairwise optimal weight realization-acceleration technique for set-theoretic adaptive parallel subgradient projection algorithm," 2005, IEEE Transactions on Signal Processing, accepted (For its preliminary version, see: Masahiro Yukawa and Isao Yamada, "On optimality of POWER weighting technique for adaptive filtering," Technical Report of IEICE, SIP2005-7, vol. 105, no. 29, pp.3742, April 2005, http://www.comm.ss.titech.ac.jp/ masahiro/ publications.html).

[33] T. Hinamoto and S. Maekawa, "Extended theory of learning identification," Transactions of the Institute of Electrical Engineers of Japan, vol. 95, no. 10, pp. 227-234, 1975.

[34] K. Ozeki and T. Omeda, "An adaptive filtering algorithm using an orthogonal projection to an affine subspace and its properties," Transactions of IEICE, vol. 67-A, no. 5, pp. 126-132, 1984.

[35] S. Haykin, Adaptive Filter Theory, Prentice-Hall, Upper Saddle River, NJ, USA, 3rd edition, 1996.

[36] A. H. Sayed, Fundamentals of Adaptive Filtering, John Wiley \& Sons, New York, NJ, USA, 2003.

[37] P. L. Combettes, "The foundations of set theoretic estimation," Proceedings of the IEEE, vol. 81, no. 2, pp. 182-208, 1993.

[38] P. L. Combettes, "Convex set theoretic image recovery by extrapolated iterations of parallel subgradient projections," IEEE Transactions on Image Processing, vol. 6, no. 4, pp. 493-506, 1997.

[39] S. Gollamudi, S. Nagaraj, S. Kapoor, and Y. H. Huang, "Setmembership filtering and a set-membership normalized LMS algorithm with an adaptive step size," IEEE Signal Processing Letters, vol. 5, no. 5, pp. 111-114, 1998.

[40] L. Guo, A. Ekpenyong, and Y. H. Huang, "Frequency-domain adaptive filtering - a set-membership approach," in Proceedings of the 37th Asilomar Conference on Signals, Systems, and Computers, pp. 2073-2077, Pacific Grove, Calif, USA, November 2003.

[41] H. H. Bauschke and J. M. Borwein, "On projection algorithms for solving convex feasibility problems," SIAM Review, vol. 38, no. 3, pp. 367-426, 1996.

[42] N. Ogura and I. Yamada, "A deep outer approximating half space of the level set of certain quadratic functions," Journal of Nonlinear and Convex Analysis, vol. 6, no. 1, pp. 187-201, 2005.

[43] J. Nagumo and A. Noda, "A learning method for system identification," IEEE Transactions on Automatic Control, vol. 12, no. 3, pp. 282-287, 1967.

[44] B. Hassibi, A. H. Sayed, and T. Kailath, " $H^{\infty}$ optimality of the LMS algorithm," IEEE Transactions on Signal Processing, vol. 44, no. 2, pp. 267-280, 1996.

[45] M. Yukawa and I. Yamada, "Adaptive parallel subgradient projection techniques with input sliding technique for stereophonic acoustic echo cancellation," in in Proceedings of the 8th IEEE International Workshop on Acoustic Echo and Noise Control (IWAENC '01), pp. 55-58, Kyoto, Japan, September 2003.

[46] S. L. Gay, "Dynamically regularized fast RLS with application to echo cancellation," in Proceedings of the IEEE International Conference on Acoustics, Speech, and Signal Processing (ICASSP '96), vol. 2, pp. 957-960, Atlanta, Ga, USA, May 1996. 
[47] S. L. Gay and S. Tavathia, "The fast affine projection algorithm," in Proceedings of the IEEE International Conference on Acoustics, Speech, and Signal Processing (ICASSP '95), vol. 5, pp. 3023-3026, Detroit, Mich, USA, May 1995.

[48] M. Rupp, "A family of adaptive filter algorithms with decorrelating properties," IEEE Transactions on Signal Processing, vol. 46, no. 3, pp. 771-775, 1998.

[49] M. Tanaka, S. Makino, and J. Kojima, "A block exact fast affine projection algorithm," IEEE Transactions on Speech and Audio Processing, vol. 7, no. 1, pp. 79-86, 1999.

[50] C. Breining, P. Dreiseitel, E. Hänsler, et al., "Acoustic echo control. An application of very-high-order adaptive filters," IEEE Signal Processing Magazine, vol. 16, no. 4, pp. 42-69, 1999.

[51] S. G. Sankaran and A. A. (Louis) Beex, "Convergence behavior of affine projection algorithms," IEEE Transactions on Signal Processing, vol. 48, no. 4, pp. 1086-1096, 2000.

[52] J.-L. Botto and G. V. Moustakides, "Stabilizing the fast Kalman algorithms," IEEE Transactions on Acoustics, Speech, and Signal Processing, vol. 37, no. 9, pp. 1342-1348, 1989.

[53] G. V. Moustakides, "Correcting the instability due to finite precision of the fast Kalman identification algorithms," Signal Processing, vol. 18, pp. 33-42, 1989.

[54] D. T. M. Slock and T. Kailath, "Numerically stable fast transversal filters for recursive least squares adaptive filtering," IEEE Transactions on Signal Processing, vol. 39, no. 1, pp. 92$114,1991$.

[55] G.-O. Glentis, K. Berberidis, and S. Theodoridis, "A unified view-efficient least squares adaptive algorithms for FIR transversal filtering," IEEE Signal Processing Magazine, vol. 16, no. 4, pp. 13-41, 1999.

[56] S. Haykin, A. H. Sayed, J. R. Zeidler, P. Yee, and P. C. Wei, "Adaptive tracking of linear time-variant systems by extended RLS algorithms," IEEE Transactions on Signal Processing, vol. 45, no. 5, pp. 1118-1128, 1997.

[57] S.-H. Leung and C. F. So, "Gradient-based variable forgetting factor RLS algorithm in time-varying environments," IEEE Transactions on Signal Processing, vol. 53, no. 8, pp. 3141-3150, 2005.

[58] S. G. Sankaran and A. A. (Louis) Beex, "Stereophonic echo cancellation using NLMS with orthogonal correction factors," in Proceedings of the International Workshop on Acoustic Echo and Noise Control (IWAENC '99), pp. 40-43, Pocono Manor, $\mathrm{Pa}, \mathrm{USA}$, September 1999.

[59] J.-T. Lai, A.-Y. Wu, and C.-C. Yeh, "A novel multipath matrix algorithm for exact room response identification in stereo echo cancellation," in Proceedings of the IEEE Workshop on Signal Processing Systems (SIPS '03), pp. 236-240, Seoul, Korea, August 2003.

[60] A. Hirano, K. Nakayama, D. Someda, and M. Tanaka, "Stereophonic acoustic echo canceller without pre-processing," in Proceedings of the IEEE International Conference on Acoustics, Speech, and Signal Processing (ICASSP '04), vol. 4, pp. 145-148, Montreal, Canada, May 2004.

[61] A. W. H. Khong and P. A. Naylor, "Reducing inter-channel coherence in stereophonic acoustic echo cancellation using partial update adaptive filters," in Proceedings of the European Signal Processing Conference (EUSIPCO '04), pp. 405-408, Vienna, Austria, September 2004.

[62] D. R. Morgan, J. L. Hall, and J. Benesty, "Investigation of several types of nonlinearities for use in stereo acoustic echo cancellation," IEEE Transactions on Speech and Audio Processing, vol. 9, no. 6, pp. 686-696, 2001.
Masahiro Yukawa received the B.E. and M. E. degrees from Tokyo Institute of Technology in 2002 and 2004, respectively. Since 2005, he has been a Research Fellow of the Japan Society for the Promotion of Science (JSPS). He is currently pursuing the Ph.D. degree in Department of Communications and Integrated Systems at Tokyo Institute of Technology. His research interests include mathematical adaptive signal processing with applications to acoustics/communications (echo cancellation, multiple-access interference suppression in CDMA, adaptive beamforming, etc.). He is a Student Member of IEEE/IEICE.

Noriaki Murakoshi received the B.E. degree in electrical and electronic engineering from Tokyo Institute of Technology, Tokyo, Japan, in 2005. He is currently a Graduate Student of the Graduate School of Decision Science and Technology, Tokyo Institute of Technology, Tokyo, Japan. His research interests include mathematical adaptive signal processing with applications to acoustics (echo canceler, hearing aids, etc.).

Isao Yamada received the B.E. degree in computer science in 1985 from University of Tsukuba and the M.E. and Ph.D. degrees in electrical and electronic engineering from Tokyo Institute of Technology, in 1987 and 1990, respectively. In 1990, he joined the Department of Electrical and Electronic Engineering at Tokyo Institute of Technology, as a Research Associate, and became an Associate Professor there in 1994.

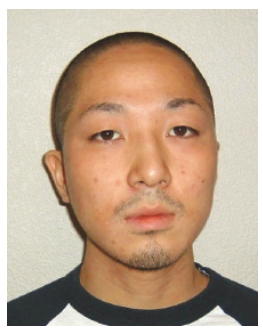
Currently, he is an Associate Professor in the Department of Communications and Integrated Systems at Tokyo Institute of Technology. He received the IEICE Excellent Paper Awards, in 1990 and 1994, and the IEICE Young Researcher Award in 1992, the ICF Research Award in 2004, and the DoCoMo Mobile Science Award in 2005 . His current research interests are in mathematical signal processing, optimization theory, and inverse problem. He is a Member of the IEEE, AMS, SIAM, JSIAM, and IEICE. He serves as an Associate Editor for the IEEE Transactions on Circuits and Systems 1 and the Journal on Multidimensional Systems and Signal Processing. 